EPJ Web of Conferences 22, 00012 (2012)

DOI: $10.1051 /$ epjconf/20122200012

(C) Owned by the authors, published by EDP Sciences, 2012

\title{
Symmetry characterization of electrons and lattice excitations
}

\author{
H. Schober \\ Institut Laue Langevin, 6 rue Jules Horowitz, 38042 Grenoble Cedex 9, France, and \\ Université Joseph Fourier, UFR de Physique, 38041 Grenoble Cedex 9, France
}

\begin{abstract}
Symmetry concerns all aspects of a physical system from the electronic orbitals to structural and magnetic excitations. In this article we will try to elaborate the fundamental connection between symmetry and excitations. As excitations are manyfold in physical systems it is impossible to treat them exhaustively. We thus concentrate on the two topics of Bloch electrons and phonons. These two examples are complementary in the sense that Bloch electrons describe single particles in an external periodic potential while phonons exemplify a decoupled system of interacting particles. The way we develop the argument gives as by-product a short account of molecular orbitals and molecular vibrations.
\end{abstract}

\section{INTRODUCTION}

In this short article we would like to give a brief account of how symmetry helps us to study excitations in solids. While the main focus will be placed on the study of lattice vibrations we will, for the sake of comparison, equally mention the Bloch states of the electron. It is our intention to make the reader familiar with the principal concepts. For a more rigorous and fully exhaustive treatment of symmetry applied to the study of electronic states and lattice excitations in molecules and crystals the reader is referred to the literature [1-5]. Basic introductions are given in most text books on solid sate physics [6-8].

We take as point of departure ideal, i.e. defect-free crystalline solids in the fully relaxed state. In such solids the atoms occupy stable average positions on a periodic lattice. We equally will briefly touch molecular systems as they allow for a more direct illustration of the concepts.

It is the task of crystallography to characterize the crystal symmetry via the 230 space groups. We thus take the knowledge of the space group as given and ask ourselves the question what information we can extract from it to facilitate the study of the crystal excitations. In order to do so we first have to identify the nature of excitations. As we will realize, symmetry gives the most important contribution at this stage of the problem, leading to the concepts of electronic band structures and phonon modes. It is assumed that the reader is familiar with the basic tools of group theory and in particular representations as exposed in the other chapters of this book.

\section{SEPARATION OF ELECTRONIC AND IONIC DEGREES OF FREEDOM}

Any solid is mathematically described by the Schrödinger equation

$$
i \hbar \frac{\partial}{\partial t} \Psi\left(\left\{\vec{R}_{I}\right\},\left\{\vec{r}_{i}\right\} ; t\right)=H \Psi\left(\left\{\vec{R}_{I}\right\},\left\{\vec{r}_{i}\right\} ; t\right) .
$$

All the information is contained in the wavefunctions $\Psi\left(\left\{\vec{R}_{I}\right\},\left\{\vec{r}_{i}\right\} ; t\right)$ that have to be weighted with their respective probabilities in the case of thermodynamic ensembles. Eq. (2.1) contains both the ionic $\left\{\vec{R}_{I}\right\}$

This is an Open Access article distributed under the terms of the Creative Commons Attribution-Noncommercial License 3.0, which permits unrestricted use, distribution, and reproduction in any noncommercial medium, provided the original work is properly cited. 
and the electronic $\left\{\vec{r}_{i}\right\}$ degrees of freedom as dynamical variables. The Hamilton operator $H$ has the form

$$
\begin{aligned}
H & =-\sum_{i} \frac{\hbar^{2}}{2 m_{i}} \nabla_{i}^{2}-\sum_{I} \frac{\hbar^{2}}{2 M_{I}} \nabla_{I}^{2}+\sum_{i \leq j} \frac{e^{2}}{\left|\vec{r}_{i}-\vec{r}_{j}\right|}+\sum_{I \leq J} \frac{Z_{I} Z_{J} e^{2}}{\left|\vec{R}_{I}-\vec{R}_{J}\right|}-\sum_{i, I} \frac{Z_{I} e^{2}}{\left|\vec{r}_{i}-\vec{R}_{I}\right|} \\
& =T_{i}+T_{I}+V_{i, j}+V_{I, J}+V_{i, I} .
\end{aligned}
$$

$T_{i}$ represents the kinetic energy of the electrons, $T_{I}$ the kinetic energy of the ions, $V_{i, j}$ the interaction among electrons, $V_{I, J}$ the interaction among ions, and $V_{i, I}$ the interaction of electrons with ions. The stationary solutions of the Schrödinger equation are given by the eigenfunctions of the Hamiltonian

$$
H \Psi_{n}\left(\left\{\vec{R}_{I}\right\},\left\{\vec{r}_{i}\right\}\right)=E_{n} \Psi_{n}\left(\left\{\vec{R}_{I}\right\},\left\{\vec{r}_{i}\right\}\right) .
$$

The eigenvalues $E_{n}$ denote the energies of the excited states. Finding the exact eigenfunctions for a solid is basically an impossible task, even if we resigne ourselves to deal only with the electronic system. Fortunately this knowledge is in general not required. A good insight into the physics of solids can be obtained with the help of a few simplifying assumptions. ${ }^{1}$ One such assumption is the adiabatic approximation. The electrons are generally a lot more mobile than the ions. They can thus be assumed to adapt immediately to any change of position in the ionic system. ${ }^{2}$ We thus can factorize the wave function into two parts

$$
\Psi\left(\left\{\vec{R}_{I}\right\},\left\{\vec{r}_{i}\right\}\right)=\psi\left(\left\{\vec{r}_{i}\right\} ;\left\{\vec{R}_{I}\right\}\right) \Phi\left(\left\{\vec{R}_{I}\right\}\right)
$$

with the electronic part satisfying

$$
\left(T_{i}+V_{i, j}+V_{i, I}\right) \psi\left(\left\{\vec{r}_{i}\right\} ;\left\{\vec{R}_{I}\right\}\right)=E_{\mathrm{el}}\left(\left\{\vec{R}_{I}\right\}\right) \psi\left(\left\{\vec{r}_{i}\right\} ;\left\{\vec{R}_{I}\right\}\right) .
$$

In this equation the ionic positions are no longer dynamic variables but simple parameters. In other words, we have effectively decoupled the electronic and ionic motion. The ionic motion itself is determined via

$$
\left(T_{I}+E_{\mathrm{el}}\left(\left\{\vec{R}_{I}\right\}\right)+V_{I, J}\right) \Phi\left(\left\{\vec{R}_{I}\right\}\right)=E \Phi\left(\left\{\vec{R}_{I}\right\}\right) .
$$

In this equation the electronic energy $E_{\mathrm{el}}\left(\left\{\vec{R}_{I}\right\}\right)$ plays the role of an external potential. The adiabatic approximation generally works very well for most materials. It should, however, be pointed out that this approximation is far from trivial and that very interesting physical phenomena arise when it breaks down.

\subsection{Symmetry in a quantum mechanical system}

We here resume very briefly the connection of symmetry with quantum mechanics as needed for the following sections. A coordinate transformation $G_{a}$ acts on the space variables $\left(\vec{r}_{1}, \ldots, \vec{r}_{N}\right)$ of the particles. ${ }^{3}$ They thus can be represented by operators $T\left(G_{a}\right)$ in the Hilbert space of the wave functions

$$
T\left(G_{a}\right) \psi\left(\vec{r}_{1}, \ldots, \vec{r}_{N}\right)=\psi^{\prime}\left(\vec{r}_{1}, \ldots, \vec{r}_{N}\right)=\psi\left(G_{a}^{-1} \vec{r}_{1}, \ldots, G_{a}^{-1} \vec{r}_{N}\right)
$$

Instead of transforming the wave functions we may equally well transform the operators in Hilbert space. In particular we may define the transformed Hamiltonian

$$
H^{\prime}=T\left(G_{a}\right) H T^{-1}\left(G_{a}\right) \text {. }
$$

\footnotetext{
1 A detailed discussion of these assumptions can be found in any standard text book on solid state physics [7].

${ }^{2}$ We denote with ions the nuclei together with the strongly bound core electrons.

${ }^{3}$ In order not to render the formalism too heavy we here do not explicitly distinguish between ionic and electronic variables. For simplicity we may equally just consider the electronic system knowing that the same arguments apply to the ions.
} 


\section{Contribution of Symmetries in Condensed Matter}

A coordinate transformation preserves the quantum mechanical system if it leaves the Hamiltonian unchanged

$$
T\left(G_{a}\right) H T^{-1}\left(G_{a}\right)=H .
$$

This equation expresses the fact that the effect of a change of the coordinate system on a function corresponds to keeping the functional form and back-transforming the coordinates. By multiplying 2.9 on the right by $T$ this condition takes the equivalent form of

$$
T\left(G_{a}\right) H=H T\left(G_{a}\right)
$$

or

$$
\left[T\left(G_{a}\right), H\right]=0 .
$$

This implies that symmetry operators commute with the Hamiltonian. It is straight-forward to show that symmetry operators form a mathematical group. Coordinate transformations like translations, rotations, reflections, inversion, etc., leave the kinetic energy unchanged. They thus are symmetry elements of the system if they preserve the potential

$$
V\left(\vec{r}_{1}, \ldots, \vec{r}_{N}\right)=V\left(G_{a}^{-1} \vec{r}_{1}, \ldots, G_{a}^{-1} \vec{r}_{N}\right)
$$

All the knowledge we can obtain on the physical system is contained in the wave functions that can be expressed in the basis of the eigenfuntions of the Hamiltonian. The presence of symmetry elements constrains the form that the eigenfunctions can assume. It is a mathematical fact that all eigenfunctions with the same energy $E$ form a subspace $V$ of Hilbert space. As we can easily show this subspace is invariant under the action of the symmetry operators. Let $\psi$ denote a wavefunction with eigenvalue $E$. Then

$$
H \psi^{\prime}=H T\left(G_{a}\right) \psi=T\left(G_{a}\right) H \psi=E T\left(G_{a}\right) \psi=E \psi^{\prime}
$$

shows that the transformed wavefunction $\psi^{\prime}$ is an eigenfunction to the same eigenvalue, where we have exploited the fact (eq. (2.11)) that symmetry operators commute with the Hamiltonian. This implies that the transformations $T\left(G_{a}\right)$ form within the subspace $V$ a representation of the symmetry group. The eigenfunctions form a basis of that representation. If this representation is not already irreducible then it can be reduced into its irreducible constituents. It is, therefore, possible to label the eigenfunctions according to the irreducible representations of the symmetry group. The presence of an irreducible representation with dimension greater than one in the decomposition of the Hilbert space introduces a corresponding degeneracy of eigenvalues.

\subsection{Equivalence}

We will now introduce the concept of equivalence, which is of central importance for the following considerations. The symmetries that we are concerned with relate to the structure. They thus establish equivalence between certain atomic sites. All symmetry elements are fully characterized if we know how they map the atoms onto each other.

The mapping of equivalent sites allows us to define a canonical representation of the symmetry group (see chapter by Canals and Schober). We consider each atomic site as a basis vector in a $N$-dimensional vector space. For the atom labelled by the index $j$ this vector $\vec{\xi}_{j}$ is chosen to have the form

$$
\vec{\xi}_{j}=\left(\xi_{1}=0 \ldots \xi_{j}=1 \ldots \xi_{N}=0\right) \text {. }
$$

The $N \times N$-matrices that describe how the vectors $\vec{\xi}_{j},(j=1, \ldots, N)$ transform under a symmetry operation form a representation of the symmetry group. This representation is called the equivalence or atomic site representation $\Gamma_{\text {a.s. }}$. 


\section{EPJ Web of Conferences}

Table 1. Character table for the group $C_{1 h}$ of the linear diatomic homopolar molecule.

\begin{tabular}{|l|l|r|l|}
\hline$\Gamma$ & $\mathrm{E}$ & $m$ & \\
\hline$A^{\prime}$ & 1 & 1 & \\
$A^{\prime \prime}$ & 1 & -1 & \\
$\chi_{\text {a.s. }}$ & 2 & 0 & $\rightarrow A^{\prime}+A^{\prime \prime}$ \\
\hline
\end{tabular}

As the mapping of the atoms onto each other is unique (every atom is mapped uniquely onto another atom) the matrixes of the equivalence representation feature the entry " 1 " exactly once per column and line. All other entries are zero. The identity operation maps every atom onto itself and thus has all the " 1 " entries in the diagonal. The character of the equivalence representation $\Gamma_{\text {a.s. }}$ for the identity is thus equal to the number of atoms. For the other classes of symmetry operations the character of $\Gamma_{\text {a.s. }}$ is identical to the number of atoms that are mapped onto themselves by the operations of that class.

The simplest example for equivalence symmetry is provided by a homopolar diatomic molecule. In the absence of an external perturbation it is evident that the two atoms forming the molecule have to be equivalent. For the purpose of illustration it is sufficient to consider the molecule as one-dimensional, i.e. we can in particular ignore the continuous rotation about the molecular axis. Under this approximation the symmetry is defined by the identity operation $E$ and a mirror plane operation $m$. The atomic site basis vectors are given by

$$
\vec{\xi}_{1}=\left(\begin{array}{l}
1 \\
0
\end{array}\right) \quad \text { and } \quad \vec{\xi}_{2}=\left(\begin{array}{l}
0 \\
1
\end{array}\right) .
$$

The equivalence representation is in this basis described by the matrices

$$
\Gamma_{\text {a.s. }}(E)=\left(\begin{array}{ll}
1 & 0 \\
0 & 1
\end{array}\right) \quad \text { and } \quad \Gamma_{\text {a.s. }}(m)=\left(\begin{array}{ll}
0 & 1 \\
1 & 0
\end{array}\right) .
$$

The equivalence representation is in general not irreducible. From inspection of the character table of the group $C_{1 h}$ (see Table 1) we deduce that the equivalence representation decomposes into two onedimensional representations

$$
\Gamma_{\text {a.s. }} \rightarrow A^{\prime}+A^{\prime \prime} \text {. }
$$

The atomic sites do not define the physical state of the system. This is provided by the wave functions in Hilbert space. The equivalence representation is thus in general not acting in the physical space. The equivalence transformations, however, leave the Hamiltonian of the system invariant. This entails, as we have seen in the preceding section, that the eigenfunctions of the Hamiltonian have to transform according to the irreducible representations of the symmetry group. ${ }^{4}$ We can create a basis for a representation of the symmetry group in Hilbert space by decorating the atomic sites with atomic orbitals (AOs). We choose these orbitals to correspond to free atoms. They thus possess full rotational symmetry and therefore, transform like scalars under the point group operations of a molecule. As we will see later in the context of vibrations this is essential if we want to avoid working with a direct product of the representation of the orbital with that of the sites.

To obtain an AO basis for our example we choose an AO on atom one that we denote as $\psi(1)$. The atomic orbital $\psi(2)$ is obtained via the mirror operation: $\psi(2)=T(m) \psi(1)$. We have learned from group theory that the symmetry adapted parts of a wave function can be obtained with the help of projection operators. These are given for our one-dimensional irreducible representations by the expression

$$
P_{\Gamma_{i}}=\frac{1}{2} \sum_{R=E, m} \chi_{\Gamma_{i}}(R) T(R) .
$$

\footnotetext{
${ }^{4}$ A mathematical version of this statement is given in eq. (6.2).
} 


\section{Contribution of Symmetries in Condensed Matter}

For $\Gamma_{i}=A^{\prime}$ we thus project out the function

$$
\psi_{\text {bonding }}=P_{A^{\prime}} \psi(1)=\frac{1}{2}[T(E) \psi(1)+T(m) \psi(1)]=\frac{1}{2}[\psi(1)+\psi(2)] .
$$

We are dealing with the well-known bonding orbital within the linear combination of atomic orbitals (LCAO) basis. The anti-bonding LCAO is obtained by projecting the atomic orbital $\psi(1)$ on the second irreducible representation $A^{\prime \prime}$

$$
\psi_{\text {anti-bonding }}=P_{A^{\prime \prime}} \psi(1)=\frac{1}{2}[T(E) \psi(1)-T(m) \psi(1)]=\frac{1}{2}[\psi(1)-\psi(2)] .
$$

While the obtained orbitals possess the symmetries that we require for eigenfunctions of the Hamiltonian we know that they actually are not eigenfunctions. The reason is connected to the fact that the basis that we have chosen is very restricted and thus not fully spanning the Hilbert space of the molecular orbitals.

Even within our restricted subspace symmetry cannot tell us which combination is bonding and which combination is not. To determine the energetic ordering of the symmetry adapted orbitals we have to use either physical intuition (the bonding orbital concentrates the charge cloud between the atoms) or calculate

$$
E_{i}=<\psi_{i}|H| \psi_{i}>
$$

for the two states.

The main advantage of symmetry is that it places constraints on the eigenfunctions. These constraints considerably reduce the calculation effort by block diagonalizing the eigenvalue problem. Let e.g. $\left\{\mid \psi_{i}>\right\}$ be an extended basis of AOs. Solving the stationary Schrödinger equation then would correspond to solving a large secular equation of the form

$$
\left|<\psi_{i}\right| H\left|\psi_{j}>-E \delta_{i, j}\right|=0 .
$$

If instead of the $\left\{\mid \psi_{i}>\right\}$ we use a corresponding set of symmetry adapted functions, obtained e.g. with the help of projection operators, then we know right from the beginning that there is no coupling between functions transforming according to different irreducible representations. The corresponding matrix elements thus vanish and the secular equation breaks up into block diagonal form. In our simple example we know thus that $<\psi_{\text {anti-bonding }}|H| \psi_{\text {bonding }}>=0$ even without writing down the Hamiltonian. This breaking up into block diagonal form is at the heart of all the considerations that will follow.

\subsection{Periodicity in crystals}

After this general introduction we will now turn our attention to crystalline systems. The characteristic property of crystals is periodicity. The crystal structure is fully described ${ }^{5}$ by a basis and the three primitive vectors $\vec{a}_{i}(i=1,2,3)$. The primitive vectors define the full set of lattice translations

$$
\vec{l}=l_{1} \vec{a}_{1}+l_{2} \vec{a}_{2}+l_{3} \vec{a}_{3}, \quad\left(l_{1}=1, \ldots, N_{1}, l_{2}=1, \ldots, N_{2}, l_{3}=1, \ldots, N_{3}\right) .
$$

The lattice translations form a sub-group of the space group of the crystal. As all symmetry operators $G(\vec{l})$ commute with each other

$$
G\left(\vec{l}_{m}\right) G\left(\vec{l}_{n}\right)=G\left(\vec{l}_{m}+\vec{l}_{n}\right)=G\left(\vec{l}_{n}\right) G\left(\vec{l}_{m}\right)
$$

the group of proper translations is Abelian. This implies that every symmetry element $G\left(\vec{l}_{m}\right)$ forms a class of its own and that all irreducible representations are one-dimensional. To deal with the finite extension of the crystal while maintaining perfect periodicity we introduce periodic boundary conditions. Translations by $L_{i}=N_{i} \vec{a}_{i},(i=1,2,3)$ are thus supposed to correspond to the identity

\footnotetext{
5 We here only deal with normal crystals. Incommensurate modulations or quasi-crystals need a special treatment.
} 
transformation. Due to the periodic boundary conditions the translations along a given primitive direction form a cyclic sub-group of the full translation group. The characters of a cyclic group, which in the case for a one-dimensional representation are identical to the representation itself, thus have to satisfy

$$
\Gamma\left(N_{i} \vec{a}_{i}\right)=\Gamma\left(\vec{a}_{i}\right)^{N_{i}}=1, \quad(i=1,2,3) .
$$

The characters of the primitive translations are thus the respective $N_{i}$ roots of unity

$$
\Gamma\left(\vec{a}_{i}\right)=\exp \left(i k_{i} a_{i}\right), \quad(i=1,2,3)
$$

with

$$
k_{i}=m_{i} \frac{2 \pi}{N_{i} a_{i}}=m_{i} \frac{2 \pi}{L_{i}} .
$$

An arbitrary translation can be expressed as a series of commuting primitive translations. We, therefore, obtain for the corresponding characters

$$
\Gamma_{\left(k_{1}, k_{2}, k_{3}\right)}(\vec{l})=\exp \left(2 \pi i\left(l_{1} k_{1}+l_{2} k_{2}+l_{3} k_{3}\right)\right) .
$$

The one-dimensional representations are, therefore, sets of phase factors, one phase factor for each translation. Each representation is labelled by the triple $\left(k_{1}, k_{2}, k_{3}\right)$. While the $l_{i}$ are the components of a vector this is not yet the case for the $k_{i}$. The notation can, however, be rendered more compact by introducing the reciprocal lattice vectors

$$
\begin{aligned}
& \vec{b}_{1}=2 \pi \frac{\vec{a}_{2} \times \vec{a}_{3}}{\vec{a}_{1} \cdot\left(\vec{a}_{2} \times \vec{a}_{3}\right)}, \\
& \vec{b}_{2}=2 \pi \frac{\vec{a}_{3} \times \vec{a}_{1}}{\vec{a}_{1} \cdot\left(\vec{a}_{2} \times \vec{a}_{3}\right)}, \\
& \vec{b}_{3}=2 \pi \frac{\vec{a}_{1} \times \vec{a}_{2}}{\vec{a}_{1} \cdot\left(\vec{a}_{2} \times \vec{a}_{3}\right)} .
\end{aligned}
$$

It is easy to see that this allows to write

$$
\Gamma_{\vec{k}}(\vec{l})=\exp (i \vec{k} \cdot \vec{l})
$$

with

$$
\vec{k}=k_{1} \vec{b}_{1}+k_{2} \vec{b}_{2}+k_{3} \vec{b}_{3} .
$$

There cannot be more irreducible representations than there are symmetry elements in the group of proper translations. To obtain a unique labeling the $m_{i}(i=1,2,3)$ in eq. (2.25) are chosen such that the vectors $\vec{k}$ lie in the first Brillouin zone. We would like to stress at this point that by using only arguments of group theory we have introduced the reciprocal space and the Brillouin zone.

\section{BLOCH ELECTRONS}

We now come back to the investigation of excitations in crystals. We will first deal with the electronic system. As we are unable to solve the system of coupled equations we make some drastic approximations. We consider first that the motion of the ions is a small perturbation for the electronic system. ${ }^{6}$ We, therefore, consider the ionic positions as fixed. In a second step we assume that the

\footnotetext{
6 This may seem justified by the energy scales. Ionic motion leads to excitations that are typically in the range from 0 to a few hundred meV while electronic band gaps often exceed several eV. However, the coupling of the electrons to the ionic motion has important consequences for transport properties. In metals it is responsible for resistance but may equally lead to superconductivity.
} 


\section{Contribution of Symmetries in Condensed Matter}

interactions between the electrons can be absorbed effectively in a one-particle potential. With these assumptions eq. (2.5), which determines the electronic wave function in the adiabatic approximation, simplifies to

$$
\left(-\frac{\hbar^{2}}{2 m} \nabla_{i}^{2}+V\left(\vec{r} ; \vec{R}_{I}^{0}\right)\right) \psi(\vec{r})=E_{\mathrm{el}} \psi(\vec{r}) .
$$

The potential $V\left(\vec{r} ; \vec{R}_{I}^{0}\right)$ in this one-electron equation is parametrized by the equilibrium positions $\vec{R}_{I}^{0}$ of the ions. It thus possesses the full symmetry of the crystal. It is in particular invariant with respect to the group of proper translations. We thus can deduce that the eigenfunctions $\psi(\vec{r})$ have to transform according to the irreducible representations of that group. According to eq. $(2.30)$ the $\psi_{\vec{k}}(\vec{r})$ will acquire a phase factor when transformed by a lattice translation

$$
\psi_{\vec{k}}(\vec{r}+\vec{l})=\exp (i \vec{k} \cdot \vec{l}) \psi_{\vec{k}}(\vec{r}), \quad \vec{k} \in \text { first } B Z .
$$

We can reformulate this statement by expressing the eigenfunctions as

$$
\psi_{\vec{k}}(\vec{r})=\exp (i \vec{k} \cdot \vec{l}) u_{\vec{k}}(\vec{r}) .
$$

Eq. (3.2) then implies that the function $u_{\vec{k}}(\vec{r})$ has the periodicity of the lattice

$$
u_{\vec{k}}(\vec{r}+\vec{l})=u_{\vec{k}}(\vec{r})
$$

This statement is known as the Bloch theorem [9]. For every value of $\vec{k}$ we have to solve the eigenvalue equation

$$
\left(\frac{\hbar^{2}}{2 m}\left(\frac{1}{i} \vec{\nabla}+\vec{k}\right)^{2}+V\left(\vec{r} ; R_{I}^{0}\right)\right) u_{\vec{k}}(\vec{r})=E_{\mathrm{el}} u_{\vec{k}}(\vec{r}) .
$$

As the problem is confined to the volume of the primitive cell the bound states will show for every $\vec{k}$ a discrete spectrum. As the Hilbert space is infinite in dimension the multiplicity of a given irreducible representation $\vec{k}$ is equally infinite. We thus can label the wave functions and eigenvalues as $\psi_{n, \vec{k}}(\vec{r})$ and $E_{n, \vec{k}}$, respectively. As $\vec{k}$ in eq. (3.5) figures as a quasi-continuous parameter $E_{n, \vec{k}}$ will change continuously with the wave vector $\vec{k}$. This leads us to the well-known band structure of crystalline solids. Respecting the Pauli principle for fermions the single-electron states $E_{n, \vec{k}}$ are filled up in order of increasing energy with 2 electrons each, one for spin up and one for spin down. This gives the ground state of the electronic system and defines the Fermi level. For insulators the filled bands (conduction bands) are separated from the empty bands (valence bands) by an energy gap. In metals conduction and valence bands connect at the Fermi surface. Excited states are obtained by displacing electrons from the valence to the conduction bands. This creates holes in the Fermi sea that will interact with the excited electrons. An example for a calculated band structure is given in figure 1.

The Bloch functions are basis functions of the translation symmetry operators in the Hilbert space of the electrons in the same way the spherical harmonic functions are basis functions of the free rotation group of an isolated atom. As all irreducible representations are one-dimensional an Abalian group does not introduce degeneracies among eigenvalues of the Hamiltonian. For this we need the extra symmetries connected with the point group operations and the improper translations of the crystal.

\section{VIBRATIONS OF THE IONIC SYSTEM}

For the following we assume to possess a sufficient knowledge of the electronic energy $E_{\mathrm{el}}\left(\left\{\vec{R}_{I}\right\}\right)$ as a function of the ionic positions to tackle the vibrational problem. For systems with reasonable unit cell size $E_{\text {el }}\left(\left\{\vec{R}_{I}\right\}\right)$ can e.g. be obtained using density-functional methods. The ionic motion is governed by 

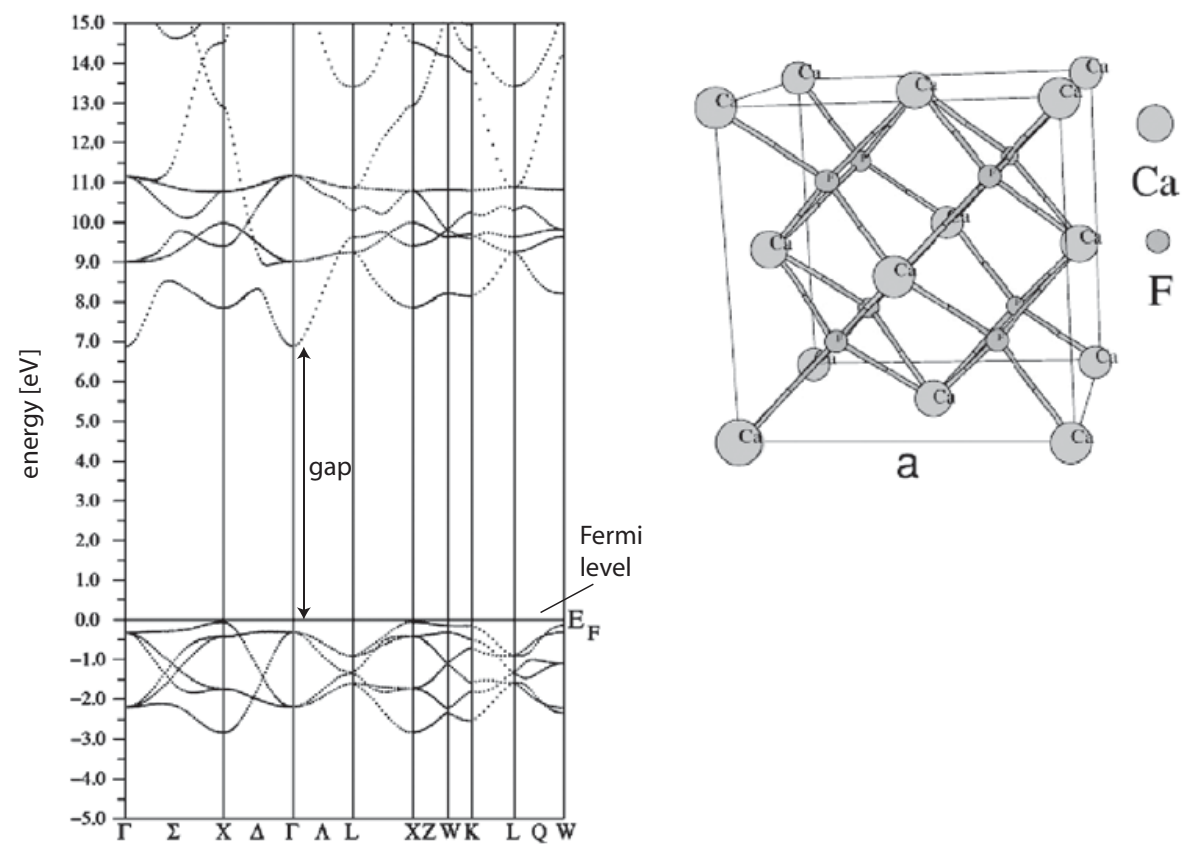

Figure 1. Calculated band structure for the super-ionic conductor $\mathrm{CaF}_{2}$ [11]. The conduction bands are separated from the valence bands by an energy gap. The symbols along the abscissa correspond to high-symmetry points of the Brillouin zone of the $\mathrm{CaF}_{2}$ structure.

eq. (2.6). The central ingredient of this equation is the potential

$$
U\left(\vec{R}_{1}, \ldots \vec{R}_{N}\right)=E_{\mathrm{el}}\left(\left\{\vec{R}_{I}\right\}\right)+V_{I, J},
$$

which contains the direct ion-ion interaction plus the electronic potential. It should be pointed out that it is the electronic part that stabilizes the solid. Investigating the lattice motion thus indirectly probes the electronic system.

$U\left(\vec{R}_{1}, \ldots \vec{R}_{N}\right)$ possesses the symmetry of the crystal. Like in the case of the electrons we can exploit this symmetry to deduce that the eigenfunctions have to transform according to the irreducible representations of the group of proper translations. Therefore,

$$
\psi\left(\vec{R}_{1}, \ldots, \vec{R}_{N}\right)=e^{i \vec{k} \cdot \vec{l}} \psi\left(\vec{R}_{1}-\vec{l}, \ldots, \vec{R}_{N}-\vec{l}\right) .
$$

In analogy to the electronic problem the translation introduces a phase factor that is fully defined by the wave vector $\vec{k}$. As we are, however, dealing with a many particle problem translational symmetry does, at that point, not allow us to constrain the form of the wave-function any further.

\subsection{Harmonic approximation}

For a general potential it is not possible to solve the many-body problem posed by eq. (2.6). An approximate solution can be found when the displacements of the ions are small with respect to their equilibrium positions $\vec{R}_{n}^{0}, n=1, \ldots N$. In this case we may expand the potential

$$
U\left(\vec{R}_{1}, \ldots \vec{R}_{N}\right)=E_{\mathrm{el}}\left(\left\{\vec{R}_{I}\right\}\right)+V_{I, J}
$$

in terms of the relative displacements

$$
\vec{u}(n \mid t)=\vec{R}_{n}(t)-\vec{R}_{n}^{0}, \quad n=1, \ldots N
$$




\section{Contribution of Symmetries in Condensed Matter}

of the ions and retain only the leading terms up to second order

$$
U\left(\vec{R}_{1}, \ldots \vec{R}_{N}\right)=U_{0}+\left.\sum_{\alpha, n=1}^{3, N} \frac{\partial U}{\partial u_{\alpha}(n)}\right|_{0} u_{\alpha}(n)+\left.\frac{1}{2} \sum_{\alpha, n=1}^{3, N} \sum_{\beta, n^{\prime}=1}^{3, N} \frac{\partial^{2} U}{\partial u_{\alpha}(n) \partial u_{\beta}\left(n^{\prime}\right)}\right|_{0} u_{\alpha}(n) u_{\beta}\left(n^{\prime}\right)
$$

$\alpha, \beta=1,2$ or 3 refer to the three space directions. The derivatives are to be taken with respect to the equilibrium positions. ${ }^{7}$ The first term in eq. (4.5) is a constant that has no influence on the dynamics. The second term corresponds to the classical forces acting upon the ions. This term should be zero in equilibrium. If the system is brought out of equilibrium, e.g. by a sudden change of temperature or pressure this term will drive the structural relaxation that brings the system back to equilibrium. The term that is important for the vibrational dynamics is the one that is quadratic in the displacements. By restricting ourselves to this term we work in the harmonic approximation. The problem of the ionic motion has thus boiled down to finding the eigenfunctions

$$
\psi_{I}\left(\vec{R}_{1}, \ldots \vec{R}_{N}\right)
$$

of the Hamiltonian

$$
\mathbf{H}=-\sum_{I} \frac{\hbar^{2}}{2 M_{I}} \vec{\nabla}_{I}^{2}+U\left(\vec{R}_{1}, \ldots \vec{R}_{N}\right)
$$

with the harmonic potential

$$
U\left(\vec{R}_{1}, \ldots \vec{R}_{N}\right)=\left.\frac{1}{2} \sum_{\alpha, n=1}^{3, N} \sum_{\beta, n^{\prime}=1}^{3, N} \frac{\partial^{2} U}{\partial u_{\alpha}(n) \partial u_{\beta}\left(n^{\prime}\right)}\right|_{0} u_{\alpha}(n) u_{\beta}\left(n^{\prime}\right)
$$

\subsection{The dynamical matrix in direct space}

The eigenvalue problem is cast into matrix notation. This considerably simplifies the formalism. The second derivatives

$$
\Phi_{\alpha \beta}\left(n, n^{\prime}\right)=-\left.\frac{\partial^{2} U}{\partial u_{\alpha}(n) \partial u_{\beta}\left(n^{\prime}\right)}\right|_{0}
$$

are called the force constants of the material. They determine the classical restoring forces acting on the ion $n$ in the direction $\vec{a}_{\alpha}$, when the ion $n^{\prime}$ is displaced along $\vec{a}_{\beta}$ (see figure 2). The force constants constitute the elements of a $(3 N \times 3 N)$-matrix $\tilde{\Phi} .^{8}$

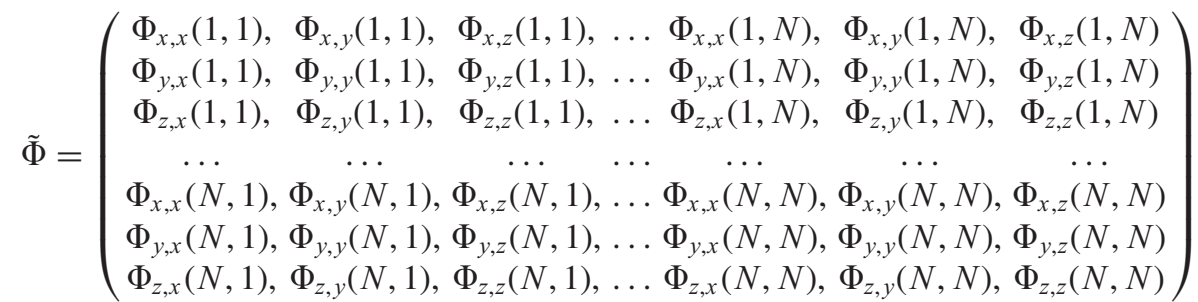

Special attention has to be given to the diagonal terms of this matrix. These so-called self-terms $\Phi_{\alpha \beta}(n, n)$ often cannot be determined directly. In that case one may use the principle of "action equals reaction"

\footnotetext{
${ }^{7}$ Due to the quantum fluctuations these positions do in general not coincide with the minima of the potential even at $T=0$. We assume that this effect can be dealt with by proper corrections.

8 We will denote the matrices by a tilde.
} 


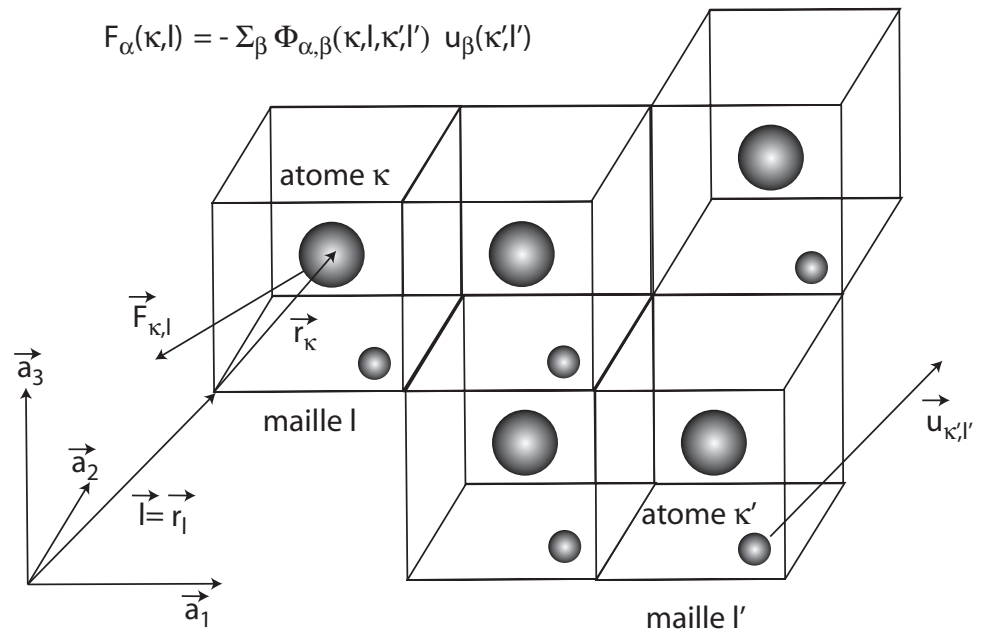

Figure 2. Schematic description of a crystal with two atoms $(\kappa=1,2)$ in the primitive cell. The displacement $\vec{u}\left(\kappa^{\prime}, \vec{l}^{\prime}\right)$ of the atome $\kappa^{\prime}$ in the cell $l^{\prime}$ creates a force acting on the atom $\kappa$ of the cell $l$. This force is given by the corresponding force constant.

to get

$$
\Phi_{\alpha \beta}(n, n)=-\sum_{n^{\prime} \neq n} \Phi_{\alpha \beta}\left(n, n^{\prime}\right)
$$

Details can be found in reference [4]. From the force constant matrix we define the dynamical matrix (in real space) via

$$
D_{\alpha \beta}\left(n, n^{\prime}\right)=\left.\frac{1}{\sqrt{M_{n} \cdot M_{n^{\prime}}}} \frac{\partial^{2} U}{\partial u_{\alpha}(n) \partial u_{\beta}\left(n^{\prime}\right)}\right|_{0}=-\frac{\Phi_{\alpha \beta}\left(n, n^{\prime}\right)}{\sqrt{M_{n} \cdot M_{n^{\prime}}}}
$$

with $M_{n}$ denoting the mass of ion $n$.

This expression simplifies in matrix notation to

$$
\tilde{D}=-\tilde{M}^{-\frac{1}{2}} \tilde{\Phi} \tilde{M}^{-\frac{1}{2}} \quad \text { with } \quad \tilde{M}_{\alpha, \beta}^{-\frac{1}{2}}\left(n, n^{\prime}\right)=\frac{1}{\sqrt{M_{n}}} \delta_{\alpha, \beta} \delta_{n, n^{\prime}} .
$$

We equally introduce a $3 \mathrm{~N}$ component vector where the displacements are weighted with the square-root of the ionic masses

$$
\overrightarrow{\bar{u}}_{3 N}=\left(\sqrt{M_{1}} \vec{u}(1), \sqrt{M_{2}} \vec{u}(2), \ldots, \sqrt{M_{N}} \vec{u}(N)\right) .
$$

It is the off-diagonal elements of the dynamical matrix that couple the ionic degrees of freedom. To achieve decoupling we have to diagonalize the dynamical matrix. To understand the underlying physics we consider the classical equations of motion

$$
M_{i} \ddot{u}_{\alpha}(n \mid t)=\sum_{\beta, n^{\prime}=1}^{3, N} \Phi_{\alpha \beta}\left(n, n^{\prime}\right) u_{\beta}\left(n^{\prime} \mid t\right),
$$

or written in the basis of weighted displacements

$$
\ddot{\bar{u}}_{\alpha}(n \mid t)=-\sum_{n^{\prime}=1, \beta=1}^{N, 3} D_{\alpha \beta}\left(n, n^{\prime}\right) \bar{u}_{\beta}\left(n^{\prime} \mid t\right) .
$$




\section{Contribution of Symmetries in Condensed Matter}

We are looking for oscillatory motion, for which

$$
\bar{u}_{\alpha}(n \mid t) \propto \cos (\omega t+\phi), \quad \forall n=1, \ldots N, \quad \forall \alpha=1,2,3,
$$

i.e. motion characterized by a frequency $\omega$ and phase $\phi$. In that case the equations of motion become

$$
\omega^{2} \overrightarrow{\bar{u}}_{3 N}=\tilde{D} \overrightarrow{\bar{u}}_{3 N} .
$$

This is a classical eigenvalue problem. The vibrational frequencies are thus to be identified with the squares of the eigenvalues of the dynamical matrix. As $\tilde{D}$ is a real symmetric matrix

$$
\tilde{D}=\tilde{D}^{T}
$$

it can be diagonalized and its $3 N$ eigenvalues are real. If the eigenvalues are positive we get real (positive) frequencies. In the case of negative eigenvalues we get imaginary frequencies corresponding to relaxational motion. This implies tha the original configuration is not stabilized by harmonic forces.

The $3 N$ eigenvectors $\vec{e}_{j}$ form a complete basis that can be chosen real and orthonormal [4]

$$
\begin{aligned}
& \vec{e}_{j} \cdot \vec{e}_{j^{\prime}}=\sum_{\alpha, n=1}^{3, N} e_{j}(\alpha, n) e_{j^{\prime}}(\alpha, n)=\delta_{j, j^{\prime}}, \\
& \sum_{j=1}^{3 N} e_{j}(\alpha, n) e_{j}\left(\beta, n^{\prime}\right)=\delta_{\alpha, \beta} \delta_{n, n^{\prime}} .
\end{aligned}
$$

Therefore, any ionic displacement can be expressed in this basis

$$
\bar{u}_{\alpha}(n)=\sum_{j=1}^{3 N} Q_{j} e_{j}(\alpha, n), \quad n=1, \ldots N, \quad \alpha=1,2,3 .
$$

The coefficients $Q_{j}$ are called the normal coordinates. A movement in which only one normal coordinate departs from zero is called a normal mode.

The variables are thus decoupled once we have found the transformation matrix that brings the dynamical matrix into diagonal form. In practical terms this amounts to finding the $3 \mathrm{~N}$-roots of the characteristic polynomial

$$
P\left(\omega^{2}\right)=\left|\left(\tilde{D}-\omega^{2} \tilde{I}_{3 N}\right)\right|
$$

where $\tilde{I}_{3 N}$ is a unit matrix of dimension $(3 N \times 3 N)$. Please not the similarity with expression 2.20.

As the transformation towards coordinates is canonical the transition to quantum mechanics can be done in the usual way by introducing the operators $\mathbf{P}_{j}$ corresponding to the conjugate momenta of the normal coordinates. The Hamilton operator then becomes diagonal and can be expressed as a sum of decoupled harmonic oscillators

$$
\mathbf{H}=\frac{1}{2}\left(\sum_{j=1}^{3 N} \mathbf{P}_{j} \cdot \mathbf{P}_{j}+\omega_{j}^{2}\left(\mathbf{Q}_{j} \cdot \mathbf{Q}_{j}\right)\right)
$$

The eigenmodes of a harmonic oscillator are readily determined. The frequency spectrum is given by

$$
E=\sum_{j=1}^{3 N}\left(n_{j}+\frac{1}{2}\right) \hbar \omega .
$$

As the quantum mechanical aspect plays no role in the following symmetry considerations we will not deepen the discussion at this point. 


\section{EPJ Web of Conferences}

\subsection{Symmetry in the vector space of ionic displacements and direct products}

We have defined the symmetry elements of a crystal as those operations that map equivalent ions onto each other (see section 2.2). The matrices describing these mappings form a $N$-dimensional representation, with $N$ the total number of atoms. This representation was called the equivalence or atomic site representation. To link with the Hilbert space of quantum mechanics we have to decorate each site either with a wave function or with a quantum mechanical operator. If the object chosen has full rotational symmetry then the equivalence representation and its decomposition can immediately be transposed to the physical space. This is for example the case if we decorate the atomic sites with s-wave functions as already outlined in section 2.2. When dealing with the vibrational problem we are interested in the displacement operators $\overrightarrow{\mathbf{u}}(l, \kappa)$. These transform like 3-dimensional vectors. The situation is analogous to decorating the atomic sites with electronic p-orbitals. A representation of the symmetry group can thus be constructed by forming the direct product of the equivalence representation with the vector representation. As only the transformation properties are important from the symmetry point of view we can safely ignore the operator character of the displacements and just consider them as classical vectors.

As direct products of representations will become important in the following discussion we would like to repeat a few facts about them. Let

$$
G_{A}=\left\{G_{a}\right\}, \quad\left(a=1, \ldots, l_{A}\right)
$$

and

$$
G_{B}=\left\{G_{b}\right\} \quad\left(b=1, \ldots, l_{B}\right)
$$

be two groups such that all operators $G_{a}$ commute with all operators $G_{b}$, i.e.

$$
G_{a} G_{b}=G_{b} G_{a}, \quad \forall a, b .
$$

Then the set of products

$$
G_{A} \otimes G_{B}=\left\{G_{a} \cdot G_{b}\right\}, \quad a=1, \ldots, l_{A}, \quad b=1, \ldots, l_{B},
$$

can be shown to form a group of order $l_{A} \cdot l_{B}$, which we call the direct product group of $G_{A}$ with $G_{B}$. Typical direct product groups are encountered in systems with inversion symmetry. In such groups $C_{i}=\{E, i\}$ forms a subgroup of the point group. In addition, both the identity operation $(E)$ and the inversion $(i)$ commute with all other point group operations. We thus can, according to our definition, consider a point group with inversion symmetry as the direct product of the point group without inversion symmetry with the group $C_{i}$. An example is the full cubic group $O_{h}$. It has a total of 48 symmetry operations. They are listed in Table 5. These operations decompose into 24 elements not involving inversion and 24 elements involving inversion. The full cubic group $O_{h}$ is thus the direct product of the group $O$ with $C_{i}$.

The direct product of two representations of $G_{A}$ and $G_{B}$ is a representation $A \otimes B$ of the direct product group. It is expressed by the well-known direct product of matrices. So if $A_{i, j}$ and $B_{k, l}$ denote the matrix elements of a representation $\Gamma_{A}$ of $G_{A}$ and $\Gamma_{B}$ of $G_{B}$, respectively, then the elements of the direct product representation $\Gamma_{A \otimes B}$ are expressed by

$$
(A \otimes B)_{i k, j l}=A_{i, j} \cdot B_{k, l}
$$




\section{Contribution of Symmetries in Condensed Matter}

This means that we decorate every entry $A_{i, j}$ of the matrix $A(m \times m)$ with the matrix $B(n \times n)$

$$
\begin{aligned}
& A \otimes B=\left(\begin{array}{c}
a_{1,1}\left(\begin{array}{ccc}
b_{1,1} & \ldots & b_{1, n} \\
\ldots & \ldots & \ldots \\
b_{n, 1} & \ldots & b_{n, n}
\end{array}\right) \\
\ldots \\
\ldots \\
a_{m, 1}\left(\begin{array}{ccc}
b_{1,1} & \ldots & b_{1, n} \\
\ldots & \ldots & \ldots \\
b_{n, 1} & \ldots & b_{n, n}
\end{array}\right)
\end{array}\right. \\
& =\left(\begin{array}{ccc}
\left(\begin{array}{ccc}
a_{1,1} b_{1,1} & \ldots & a_{1,1} b_{1, n} \\
\ldots & \ldots & \ldots \\
a_{1,1} b_{n, 1} & \ldots & a_{1,1} b_{n, n}
\end{array}\right) & \ldots & \left(\begin{array}{ccc}
a_{1, m} b_{1,1} & \ldots & a_{1, m} b_{1, n} \\
\ldots & \ldots & \ldots \\
a_{1, m} b_{n, 1} & \ldots & a_{1, m} b_{n, n}
\end{array}\right) \\
& \ldots & \\
\left(\begin{array}{ccc}
a_{m, 1} b_{1,1} & \ldots & a_{m, 1} b_{1, n} \\
\ldots & \ldots & \ldots \\
a_{m, 1} b_{n, 1} & \ldots & a_{m, 1} b_{n, n}
\end{array}\right) & \ldots & \left.\left(\begin{array}{ccc}
a_{m, m} b_{1,1} & \ldots & a_{m, m} b_{1, n} \\
\ldots & \ldots & \ldots \\
a_{m, m} b_{n, 1} & \ldots & a_{m, m} b_{n, n}
\end{array}\right)\right) .
\end{array}\right.
\end{aligned}
$$

It can be shown that the direct product $\Gamma_{\alpha} \otimes \Gamma_{\beta}$ of two irreducible representations $\Gamma_{\alpha}$ and $\Gamma_{\beta}$ of the groups $G_{A}$ and $G_{B}$, respectively, gives an irreducible representation of the direct product group $G_{A} \otimes G_{B}$, and further that all irreducible representations of $G_{A} \otimes G_{B}$ can be generated by such products.

However, attention is required in the case where the direct product is formed between a group $G_{A}$ and itself. While the direct products of the irreducible representations of $G_{A}$ are irreducible representations of $G_{A} \otimes G_{A}$ they are in general only reducible representations of $G_{A}$.

The characters of a direct product of representations are simply the products of the characters

$$
\chi^{\Gamma_{\alpha} \otimes \Gamma_{\beta}}\left(G_{A} \cdot G_{B}\right)=\chi^{\Gamma_{\alpha}}\left(G_{A}\right) \cdot \chi^{\Gamma_{\beta}}\left(G_{B}\right) .
$$

In the specific case of $G_{A}=G_{B}$ we know that $\Gamma_{\alpha} \otimes \Gamma_{\alpha^{\prime}}$ is a representation of $G_{A}$. We can use the standard formula to find the decomposition of this representation into irreducible representations of $G_{A}$. In particular the characters of $\Gamma_{\alpha} \otimes \Gamma_{\alpha^{\prime}}$ can be expressed as linear combinations of the characters of the irreducible representations of $G_{A}$

$$
\chi^{\Gamma_{\alpha} \otimes \Gamma_{\alpha}^{\prime}}\left(G_{a} \cdot G_{A}\right)=\chi^{\Gamma_{\alpha}}\left(G_{A}\right) \cdot \chi^{\Gamma_{\alpha}^{\prime}}\left(G_{A}\right)=\sum_{\mu} a_{\alpha, \alpha^{\prime}, \mu} \chi^{\Gamma_{\mu}}\left(G_{A}\right) .
$$

The coefficients $a_{\alpha, \alpha^{\prime}, \mu}$ can be calculated with the help of the character tables via

$$
a_{\alpha, \alpha^{\prime}, \mu}=\frac{1}{h} \sum_{C_{v}} N_{C_{v}} \chi^{\Gamma_{\mu}}\left(C_{v}\right)^{*}\left[\chi^{\Gamma_{\alpha}}\left(C_{v}\right) \cdot \chi^{\Gamma_{\alpha}^{\prime}}\left(C_{v}\right)\right] .
$$

$C_{v}$ represents a class of symmetry elements of $G_{A}$ and $N_{C_{v}}$ denotes the number of elements in that class. The parameter $h$ is the order of the group.

\subsection{Molecular vibrations}

To illustrate the concept (and despite the fact that we want to concentrate the discussion on crystals) let us consider a simple molecule. The formalism that we have developed in section 4.2 for finding the eigenmodes is fully applicable to the molecule as long as we stay in the harmonic approximation. The vibrations of the molecule are thus determined once we have diagonalized the dynamical matrix. 


\section{EPJ Web of Conferences}

This is an eigenvalue problem identical to the one that we have encountered in section 2.1 for the stationary Schrödinger equation. A symmetry operation preserves the potential and thus automatically the dynamical matrix. Thus the corresponding operators in the vector space of atomic displacements will commute with the dynamical matrix

$$
T\left(G_{a}\right) \tilde{D} T^{-1}\left(G_{a}\right)=\tilde{D} .
$$

From this we deduce that the eigenvectors of the dynamical matrix can be chosen to transform according to the irreducible representations of the displacement representation. Eigenvectors corresponding to the same frequency will form an invariant subspace. Symmetry can thus help us to block diagonalize the dynamical matrix. In many cases this is sufficient to fix the eigenvectors, i.e. the displacement pattern.

To illustrate how to proceed in practice let us consider the explicit example of the water molecule.

- We first have to identify the symmetry.

The symmetry of the water molecule corresponds to the point group $C_{2 v}(2 \mathrm{~mm})$. Apart from the identity operation $E$ there are three symmetry operations that include a rotation by $180^{\circ}$ around the $z$ axis, a reflection plane $\sigma_{v}$ in the plane of the molecule and another reflection plane $\sigma_{v}^{\prime}$ perpendicular to the plane of the molecule (see figure 3). Each symetry element is in a class of its own. The respective characters are summarized in table 2 .

- We have to determine the equivalence transformation and reduce it.

The identity operation maps all atoms onto themselves. It thus is represented by the matrix

$$
\left(\begin{array}{lll}
1 & 0 & 0 \\
0 & 1 & 0 \\
0 & 0 & 1
\end{array}\right)
$$

and has a character of 3 . The same holds for the reflection in the molecular plane that leaves all the atoms untouched. The rotation and the vertical reflection exchange the hydrogen atoms while leaving the oxygen atom unaffected. They are thus represented by the matrix

$$
\left(\begin{array}{lll}
1 & 0 & 0 \\
0 & 0 & 1 \\
0 & 1 & 0
\end{array}\right)
$$

where the first row and first column correspond to oxygen. The character $\Gamma_{\text {a.s. }}$ of these elements is one. With the help of the characters of the irreducible representations of the group $C_{2 v}$ we find that $\Gamma_{\text {a.s. }}$ reduces into $2 A_{1}+B_{1}$. These results are summarized in table 3 .

- Find the displacement representation.

The displacements are vectors and thus transform like $(x, y, z)$. In the coordinate system chosen (see figure 3 ) the $z$ component of the vector remains unaffected by all symmetry elements of the water molecule. $z$ thus necessarily transforms like $A_{1}$. The $x$ component is inverted by the $180^{\circ}$ rotation and by the $\sigma_{v}^{\prime}$ reflection, while it is not affected by $\sigma_{v} . x$ therefore transforms like $B_{1}$. The $y$ component changes sign under the $180^{\circ}$ rotation as well as under the $\sigma_{v}$ reflection, while it is not affected by $\sigma_{v}^{\prime} . y$ consequently transforms according to $B_{2}$. We could have deduced this result directly from the characters (Table 2). As we need all three components a vector transforms like

$$
\Gamma_{\mathrm{vec}}=A_{1}+B_{1}+B_{2} .
$$

The displacement or eigenvector representation is obtained by decorating each atomic site with a displacement vector. It is thus the direct product of the vector representation with the equivalence representation

$$
\Gamma_{\text {e.v. }}=\Gamma_{\text {a.s. }} \otimes \Gamma_{\text {vec }} .
$$




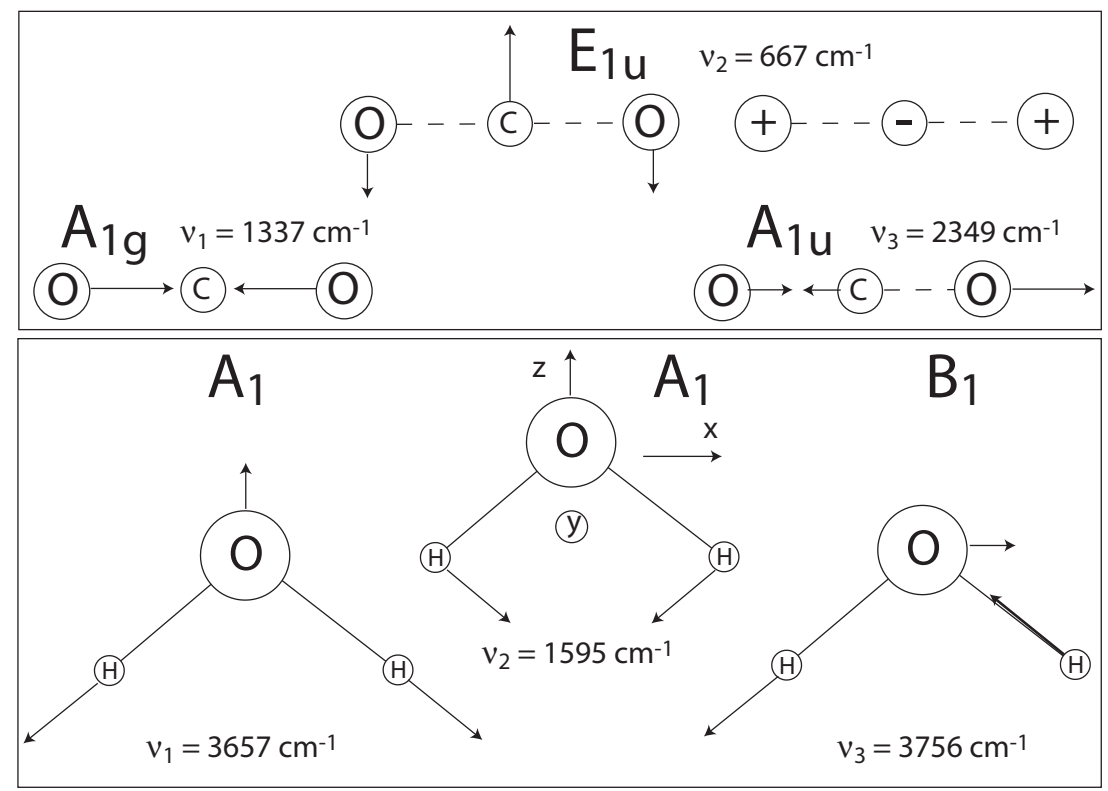

Figure 3. Normal modes for the linear $\mathrm{CO}_{2}$ and for the planar $\mathrm{H}_{2} \mathrm{O}$ molecule. The vibrations denoted by $v_{1}$ imply motions that are symmetric along the two bonds. These bond-stretching modes are high in frequency. The vibrations $v_{2}$ bend the bond angle. They are lower in frequency. The vibrations $v_{3}$ act asymmetrically on the bonds. All vibrational modes are external modes, i.e. they leave the orientation and center of gravity of the molecules invariant. All vibrations transform according to irreducible representations of the symmetry group of the molecules. For $\mathrm{H}_{2} \mathrm{O}$ these representations $\left(2 \mathrm{~A}_{1}\right.$ and $\left.\mathrm{B}_{1}\right)$ are all one-dimensional. In the case of $\mathrm{CO}_{2}$ we have two one-dimensional representations $\left(A_{1 g}\right.$ and $\left.A_{1 u}\right)$ plus a two-fold degenerate representation $\mathrm{E}_{1 u}$. The second partner of that representation has its motion perpendicular to the page. Please note that the linear molecule has only 5 external modes, due to the absence of a rotation about the molecular axis.

Table 2. Character table and common basis functions for the point group $C_{2 v}(2 \mathrm{~mm})$ (taken from [1]).

\begin{tabular}{|l|l|l|r|r|r|r|}
\hline$\Gamma$ & & & $\mathrm{E}$ & $\mathrm{C}_{2}$ & $\sigma_{v}$ & $\sigma_{v}^{\prime}$ \\
\hline $\mathrm{A}_{1}$ & $x^{2}, y^{2}, z^{2}$ & $z$ & 1 & 1 & 1 & 1 \\
$\mathrm{~A}_{2}$ & $x y$ & $R_{z}$ & 1 & 1 & -1 & -1 \\
$\mathrm{~B}_{1}$ & $x z$ & $R_{y}, x$ & 1 & -1 & 1 & -1 \\
$\mathrm{~B}_{2}$ & $y z$ & $R_{x}, y$ & 1 & -1 & -1 & 1 \\
\hline
\end{tabular}

Table 3. Characters for the equivalence or atomic site representation of the water molecule (taken from [1]).

\begin{tabular}{l|r|r|r|r|l}
\hline & $\mathrm{E}$ & $\mathrm{C}_{2}$ & $\sigma_{v}$ & $\sigma_{v}^{\prime}$ & \\
\hline$\Gamma_{\text {a.s. }}\left(\mathrm{H}_{2} \mathrm{O}\right)$ & 3 & 1 & 3 & 1 & $\rightarrow 2 \mathrm{~A}_{1}+\mathrm{B}_{1}$ \\
\hline
\end{tabular}

In the case of the water molecule

$$
\begin{aligned}
\Gamma_{\text {e.v. }} & =\left(2 A_{1}+B_{1}\right) \otimes\left(A_{1}+B_{1}+B_{2}\right) \\
& =3 A_{1}+3 B_{1}+2 B_{2}+A_{2} .
\end{aligned}
$$

To get the irreducible representation we have used the fact that the characters of a direct product of representations are the product of the characters of these representations (see Table 4) and the formula presented in section 4.3.

- Identify the eigenmodes.

A free molecule can be displaced and turned in space. This leads to 6 external modes. The symmetry of these external motions can be identified with the help of Table 2. The translations transform like a 


\section{EPJ Web of Conferences}

Table 4. Character table for the reduction of the displacement representation of the water molecule.

\begin{tabular}{|l|r|r|r|r|}
\hline$\Gamma$ & $\mathrm{E}$ & $\mathrm{C}_{2}$ & $\sigma_{v}$ & $\sigma_{v}^{\prime}$ \\
\hline $2 A_{1}+B_{1}$ & 3 & 1 & 3 & 1 \\
$A_{1}+B_{1}+B_{2}$ & 3 & -1 & 1 & 1 \\
$\left(2 A_{1}+B_{1}\right) \otimes\left(A_{1}+B_{1}+B_{2}\right)$ & 9 & -1 & 3 & 1 \\
$3 A_{1}+3 B_{1}+2 B_{2}+A_{2}$ & 9 & -1 & 3 & 1 \\
\hline
\end{tabular}

vector, i.e. like

$$
\Gamma_{\text {trans. }}=A_{1}+B_{1}+B_{2}
$$

The rotations transform like the angular momentum $\vec{r} \times \vec{p}$ that is symbolically denoted by $\left(R_{x}, R_{y}, R_{z}\right)$. From Table 2 we deduce that $\left(R_{x}, R_{y}, R_{z}\right)$ transforms according to

$$
\Gamma_{\text {rot. }}=A_{2}+B_{1}+B_{2} \text {. }
$$

This implies that the three remaining vibrations transform like

$$
\Gamma_{\text {vib. }}=2 A_{1}+B_{1} .
$$

As the irreducible representations are all one-dimensional, we conclude that from a symmetry point of view there are no degenerate vibrations in the water molecule. With rather simple arguments we can now identify the eigenvectors of the water vibrations. The two modes with $A_{1}$ symmetry must leave the molecular symmetry unchanged. This implies necessarily that the two hydrogen atoms must move in the same direction and that the oxygen ion must move along the symmetry direction. As all eigenvectors have to be orthogonal to each other the center of mass has to be preserved and we do not allow for any rotation. This immediately implies that the hydrogen motion is equally confined to the molecular plane. We are left with two orthogonal modes. The first one is a breathing mode where all atoms move away from the center of gravity. The second one is a bending mode of the $\mathrm{H}-\mathrm{O}-\mathrm{H}$ angle. The remaining mode is of $B_{1}$ symmetry. In that case the $x$ direction is inverted under the action of the rotation, which implies that the oxygen atom vibrates along $x$ and in opposition to the hydrogen atoms. The patterns of the three water vibrations are shown in figure 3 . In a similar way we can determine the vibrations of a linear molecule like $\mathrm{CO}$ equally shown in that figure.

\subsection{Ionic Bloch functions or phonons}

A crystal can be identified with a huge molecule. The procedure for constraining the eigenmodes that was outlined in the preceding section can thus be applied. As the salient feature of a crystal is the lattice periodicity we will proceed in two stages. We first study the consequences arising from the subgroup of proper translations. We will discover that - in full analogy with the Bloch electrons - the ionic displacements are periodic with the periodicity being defined by a wave vector $\vec{k}$, which is to be chosen within the first Brillouin zone. In a second step we will then investigate how the remaining symmetry elements of the space group constrain the eigenvectors for a given wave vector.

A crystal is described by a basis and a lattice. The group of proper translations is fully expressed by the lattice. Each translation maps the lattice sites onto each other. The lattice sites thus constitute a representation of the translation group, which we denote by $\Gamma_{\text {l.s. }}$. The characters of the lattice site representation are obtained by counting the number of sites that are mapped onto themselves. In the case of translations the characters of the lattice site representation are thus zero with the exception of the identity transformation, the character of which is equal to the total number of lattice sites $N=N_{1} N_{2} N_{3}$. The lattice site transformation can be reduced into the irreducible representations of the translation group. We have seen in section 2.3 that the irreducible representations of the lattice site representation 


\section{Contribution of Symmetries in Condensed Matter}

are one-dimensional and that the $(1 \times 1)$ matrices are given by the phase factors (see eq. $(2.30))$

$$
\Gamma_{\vec{k}}(\vec{l})=\exp (i \vec{k} \cdot \vec{l})
$$

In the decomposition each wave vector appears exactly once as is easily demonstrated using the wellknown identity relation

$$
\sum_{\vec{k} \in B . Z .} \exp (i \vec{k} \cdot \vec{l})=N \delta_{\vec{l}, 0} \quad \forall \quad \vec{l}=\sum_{i=1}^{3} n_{i} \vec{a}_{i}, \quad 0 \leq n_{i}<N_{i} .
$$

The transition from the lattice sites to the atomic displacements is carried out in two stages by decorating each lattice site first with the atoms of the basis and then with the respective displacement vectors. The first step leads us to the equivalence representation $\Gamma_{\text {a.s. }}$. By decorating each atomic site with a vector we arrive at the displacement representation

$$
\Gamma_{\text {displ. }}=\Gamma_{\text {vec }} \otimes \Gamma_{\text {basis }} \otimes \Gamma_{\text {l.s. }} .
$$

We have seen in section 4.4 that the eigenvectors of the atomic displacements have to transform according to the irreducible representations of the displacement representation $\Gamma_{\text {displ. }}$. Vectors are not affected by translations. If we ignore for the moment any possible point group symmetries then each vector component transforms according to the identity representation, which we denote by $A$. The three components of the vector thus simply replicate the equivalence site representation $\Gamma_{\text {a.s. }} 3 r$-times, with $r$ denoting the number of atoms in the basis. As by definition proper translations cannot mix the different atoms of the basis the displacement representation of the translation group trivially diagonalizes into $r$ different blocks.

The block diagonal form implies that the displacement eigenvectors, which are a basis of the irreducible displacement representations, transform under an arbitrary translation operation $\vec{\tau}$ like

$$
\vec{e}(\kappa, \vec{\tau}+\vec{l})=\exp (i \vec{k} \cdot \vec{\tau}) \vec{e}(\kappa, \vec{l})
$$

The index $\kappa=1, r$ labels the different atoms in the unit cell. If we take the displacements in the cell at the origin as the reference and denote $\vec{e}(\kappa, 0)$ simply by $\vec{e}(\kappa)$ then

$$
\vec{e}(\kappa, \vec{l})=\exp (i \vec{k} \cdot \vec{l}) \cdot \vec{e}(\kappa)
$$

The eigenvectors thus have the same property as the Bloch functions of the electrons (see eq. (3.3)). The displacement of an atom in the cell $\vec{l}$ is related to the displacement of an equivalent atom at the origin by a phase factor that is completely determined by the wave vector $\vec{k}$. The periodic lattice excitations are called phonons.

We may now use this result to considerably simplify the dynamical matrix. The periodicity of the lattice imposes via eq. (4.40) that the eigensolutions have the form

$$
\vec{u}(\kappa, \vec{l} \mid t)=\vec{u}(\kappa \mid \vec{k}) e^{i(\vec{k} \cdot \vec{l}-\omega t)} .
$$

The $\vec{u}(\kappa \mid \vec{k})$ are defined within the primitive cell at the origin and the wave vectors $\vec{k}$ are chosen within the first Brillouin zone.

The second derivatives

$$
\Phi_{\alpha \beta}\left(\begin{array}{ll}
\vec{l} & \vec{l}^{\prime} \\
\kappa & \kappa^{\prime}
\end{array}\right)=\left.\frac{\partial^{2} U}{\partial u_{\alpha}(\kappa, \vec{l}) \partial u_{\beta}\left(\kappa^{\prime}, \vec{l}^{\prime}\right)}\right|_{0}
$$

are the force constants that we had already encountered in section 4.2 but now expressed in a notation that is adapted to the the crystal. We again introduce a mass weighted vector of dimension $3 r N$

$$
\overrightarrow{\vec{u}}(\kappa, \vec{l} \mid t)=\sqrt{M_{\kappa}} \vec{u}(\kappa, \vec{l} \mid t)=\sqrt{M_{\kappa}} \vec{u}(\kappa \mid \vec{k}) e^{i(\vec{k} \cdot \vec{l}-\omega t)}=\overrightarrow{\vec{u}}(\kappa \mid \vec{k}) e^{i(\vec{k} \cdot \vec{l}-\omega t)} .
$$


In this notation the classical equations of motion

$$
M_{\kappa} \ddot{u}_{\alpha}(\kappa, \vec{l} \mid t)=\sum_{\beta, \kappa^{\prime}, \vec{l}^{\prime}} \Phi_{\alpha \beta}\left(\begin{array}{ll}
\vec{l} & \vec{l}^{\prime} \\
\kappa & \kappa^{\prime}
\end{array}\right) u_{\beta}\left(\kappa^{\prime}, \vec{l}^{\prime} \mid t\right)
$$

read like

$$
\ddot{\bar{u}}_{\alpha}(\kappa, \vec{l} \mid t)=-\sum_{\beta, \kappa^{\prime}, \vec{l}^{\prime}} D_{\alpha \beta}\left(\begin{array}{ll}
\vec{l} & \vec{l}^{\prime} \\
\kappa & \kappa^{\prime}
\end{array}\right) \bar{u}_{\beta}\left(\kappa^{\prime}, \vec{l}^{\prime} \mid t\right)
$$

with

$$
D_{\alpha \beta}\left(\begin{array}{ll}
\vec{l} & \vec{l}^{\prime} \\
\kappa & \kappa^{\prime}
\end{array}\right)=\frac{\Phi_{\alpha \beta}\left(\begin{array}{cc}
\vec{l} & \vec{l}^{\prime} \\
\kappa & \kappa^{\prime}
\end{array}\right)}{\sqrt{M_{\kappa} M_{\kappa^{\prime}}}} .
$$

Using the symmetry adapted expression (4.41) for $\vec{u}(\kappa, \vec{l} \mid t)$ this equation transforms to

$$
\omega^{2} \bar{u}_{\alpha}(\kappa \mid \vec{k})=\sum_{\kappa^{\prime}, \beta} D_{\alpha \beta}\left(\kappa, \kappa^{\prime} \mid \vec{k}\right) \bar{u}_{\beta}\left(\kappa^{\prime} \mid \vec{k}\right),
$$

with the dynamical matrix in reciprocal space $\tilde{D}(\vec{k})$ defined for each wave vector $\vec{k}$ of the first Brillouin zone as

$$
D_{\alpha \beta}\left(\kappa, \kappa^{\prime} \mid \vec{k}\right)=\sum_{\vec{l}-\vec{l}^{\prime}} D_{\alpha \beta}\left(\begin{array}{ll}
\vec{l} & \overrightarrow{l^{\prime}} \\
\kappa & \kappa^{\prime}
\end{array}\right) \exp \left(i \vec{k}\left(\overrightarrow{l^{\prime}}-\vec{l}\right)\right) .
$$

$\tilde{D}(\vec{k})$ can be considered the Fourier transform of the dynamical matrix in real space. Due to the periodicity of the lattice it only depends on the relative distances between the atoms.

The problem of finding the eigenvalues and eigenvectors has finally taken the form

$$
\omega_{j}^{2}(\vec{k}) \vec{e}_{j}(\vec{k})=\tilde{D}(\vec{k}) \vec{e}_{j}(\vec{k}) .
$$

We thus have to determine for every wave vector $\vec{k}$ the $3 r$ eigenvalues $\omega_{j}^{2}(\vec{k})$ of the dynamic matrix $\tilde{D}(\vec{k})$ with their associated eigenvectors $\vec{e}_{j}(\vec{k})$.

The dynamical matrix $\tilde{D}(\vec{k})$ is by definition hermitian

$$
D_{\alpha \beta}\left(\kappa, \kappa^{\prime} \mid \vec{k}\right)=D_{\beta \alpha}^{*}\left(\kappa^{\prime}, \kappa \mid \vec{k}\right) .
$$

Therefore the eigenvalues $\omega_{j}^{2}$ exist and are real. As $\tilde{D}(\vec{k})$ is in general not symmetric the eigenvectors are complex. They can be chosen to form an orthonormal basis

$$
\vec{e}_{j}^{*}(\vec{k}) \cdot \vec{e}_{j^{\prime}}(\vec{k})=\sum_{\alpha, \kappa=1}^{3, r} e_{j}^{*}(\alpha, \kappa \mid \vec{k}) e_{j^{\prime}}(\alpha, \kappa \mid \vec{k})=\delta_{j, j^{\prime}}
$$

It is a lot easier to diagonalize a matrix of dimension $3 r \times 3 r$ for several $\vec{k}$-values than to diagonalize a single matrix of dimension $3 N \times 3 N$. The fact that we only have to diagonalize the matrix $\tilde{D}(\vec{k})$ for every wave vector $\vec{k}$ constitutes thus an enormous progress with respect to a situation without symmetry.

Like in the case of the Bloch electrons the wave vector can be considered a quasi-continuous parameter. The frequencies $\omega_{j}(\vec{k})$ as a function of $\vec{k}$ thus form continuous sheets in reciprocal space. If we cut these sheets along a specific $\vec{k}$ direction then we obtain the well-known phonon dispersion curves. A concrete example will be given in section 5.6.

As the dynamical matrix in reciprocal space is of dimension $3 r$ we get for every wave vector $\vec{k}$ exactly $3 r$ phonon branches. This corresponds to the $3 r$ degrees of freedom of the atoms in the primitive 


\section{Contribution of Symmetries in Condensed Matter}

cell. Each branch gives one harmonic oscillator for a given value of $\vec{k}$. As we have a total of $N$ distinct $\vec{k}$ values in the first Brillouin zone we retrieve in total the $3 r N$ degrees of freedom of the crystal. Each eigenmode defines a normal coordinate. As we are here only concerned with aspects related to symmetry we will not go into a discussion of the quantum mechanical aspects of phonon excitations and how they influence the physical properties of the crystal. These aspects are well treated in solid state text books and specialized works on lattice dynamics $[3-5,7,8,10]$.

\section{THE SYMMETRY OF THE RECIPROCAL LATTICE}

The discussion in the previous section has shown that working with a basis that is adapted to the translational symmetry of the crystal means investigating the dynamics in reciprocal space. This follows directly from the fact that the dynamical matrix $\tilde{D}(\vec{k})$ and thus its eigenvectors $\vec{e}_{j}(\vec{k})$ and eigenfrequencies $\omega_{j}(\vec{k})$ are functions of the wave vector $\vec{k}$. The same statement applies to electronic Bloch functions. If the system possesses only lattice symmetry then the disccusion stops here. In general, a crystal features, however, more complex symmetry operations. In the Seitz notation the symmetry elements are given by

$$
G_{a}=\left\{S_{a} \mid \vec{v}\left(S_{a}\right)+\vec{l}(m)\right\},
$$

where $S_{a}$ is a rigid point group transformation (rotation, reflexion, inversion), $\vec{v}\left(S_{a}\right)$ the vector for the fractional translation that accompanies a screw-axis or glide plane and $\vec{l}(m)$ a lattice translation. For symorphic space groups without screw axes and glide planes $\vec{v}\left(S_{a}\right)$ is always zero.

The symmetry elements $S_{a}$ will act on the dynamical matrix and will thus provide a further reduction within the subspace of representations belonging to the same $\vec{k}$. They will in addition relate representations belonging to different $\vec{k}$. To exploit these additional possibilities we have to determine the action of the symmetry elements $S_{a}$ in reciprocal space.

\subsection{Transformation properties of the wave vector}

The reciprocal lattice is defined via its orthogonality with the direct lattice. This orthogonality is expressed through the scalar product (see eq. (2.29))

$$
\vec{a}_{i} \cdot \vec{b}_{j}=2 \pi \delta_{i, j}, \quad(i, j=1,2,3)
$$

and has to be preserved under symmetry operations. As scalar products are constants and thus invariant under rotations, inversions and translations they do not change if we apply the same symmetry operation to both members of the product. Therefore

$$
\left[T^{-1}\left(G_{a}\right) \vec{a}_{i}\right] \cdot b_{j}=\left[T\left(G_{a}\right) T^{-1}\left(G_{a}\right) \vec{a}_{i}\right] \cdot\left[T\left(G_{a}\right) \vec{b}_{j}\right]=\vec{a}_{i} \cdot\left[T\left(G_{a}\right) b_{j}\right], \quad \forall G_{a} .
$$

Thus the action of a symmetry element $G_{a}$ on the vectors of the direct space is the same as the action of the inverse $G_{a}^{-1}$ on the vectors of reciprocal space. As the set of inverse operations $\left\{G_{a}^{-1}\right\}$ is by definition identical to the set $\left\{G_{a}\right\}$ we can treat the reciprocal space - from a purely symmetry point of view - exactly in same the manner as the direct space.

An important concept for the following discussion is the group and the star of a wave vector $\vec{k}$. The group of a wave vector $\vec{k}$ is composed of the set of symmetry operations $\left\{G_{a}(\vec{k})\right\}$ that either leave the wave vector invariant or displace the wave vector by a reciprocal lattice vector $\vec{K}_{m}$

$$
G_{a}(\vec{k}): \vec{k} \rightarrow \vec{k}+\vec{K}_{m} .
$$

For $\vec{k}=0$ the group of the wave vector coincides trivially with the space group of the crystal. For other wave vectors the group of the wave vector forms a subgroup of the space group. If the group of the wave vector is not identical to the space group then there are symmetry elements that map $\vec{k}$ on $\vec{k}^{\prime} \neq \vec{k}$. The 
For wave vectors inside the Brillouin zone

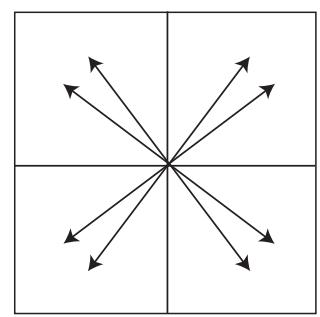

Star of 8

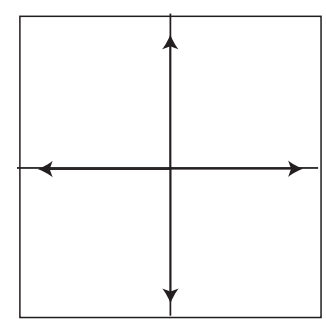

Star of 4

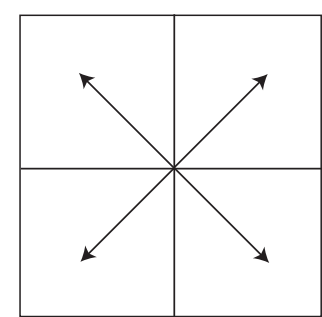

Star of 4

For wave vectors at the boundary of the Brillouin zone
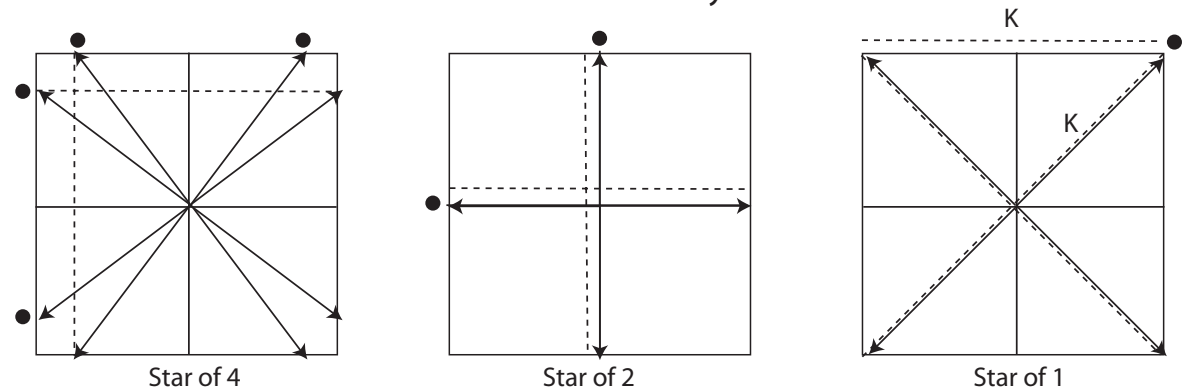

Figure 4. The star of a wave vector $\vec{k}$ is given by the set of inequivalent vectors $\left\{T\left(G_{a}\right) \vec{k}\right\}$ obtained by applying all symmetry elements $G_{a}$ to the wave vector. For a general position within the Brillouin zone the star has the dimension of the point group as every $T\left(G_{a}\right) \vec{k}$ is distinct from $\vec{k}$. For high symmetry directions (diagonals and medians of the square) subsets of symmetry operations map $\vec{k}$ onto itself. The dimension of the star is reduced. Special attention has to be given to points that lie on the zone boundary. Due to translational symmetry points that can be related by reciprocal lattice vectors $\vec{K}_{m}$ are equivalent. In the examples shown in the second row the vectors with a black dot are sufficient to form an inequivalent set. Many of the complications related to the application of group theory to space groups have their origin in the coupling of point group operations and lattice translations. (figure inspired by [1])

set of distinct $\vec{k}^{\prime}$ vectors is called the star of the vector $\vec{k}$. It is illustrated in figure 4 for the case of a planar square lattice.

The point groups of the wave vectors $\vec{k}$ are tabulated for all space groups $[12,13]$.

\subsection{The transformation properties of a Bloch function}

We have seen in section 3 that the Bloch functions form a basis for the irreducible presentations of the translation group. In the case of ionic motion the Bloch functions are the phonons. To treat the influence of space groups on electronic and ionic excitations in their totality we thus have to investigate the action of point group operations $T\left(P_{a}\right)$ on the Bloch functions. We denote the transformed Bloch function by

$$
\psi_{P_{a} \vec{k}}(\vec{r})=T\left(P_{a}\right) \psi_{\vec{k}}(\vec{r})=\exp \left(i P_{a} \vec{k} \cdot \vec{r}\right) u_{P_{a} \vec{k}}(\vec{r})
$$

Transforming the function by $T\left(P_{a}\right)$ corresponds to keeping the functional form and transforming the coordinate system in the opposite sense. Thus

$$
\psi_{P_{a} \vec{k}}(\vec{r})=\exp \left(i P_{a} \vec{k} \cdot \vec{r}\right) u_{\vec{k}}\left(P_{a}^{-1} \vec{r}\right)
$$




\section{Contribution of Symmetries in Condensed Matter}

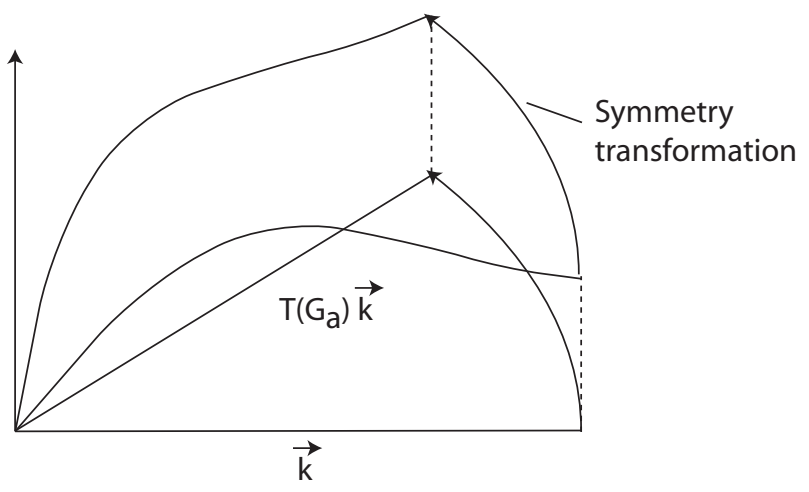

Figure 5. If a symmetry operation relates two directions in reciprocal space then the electronic and vibrational dispersion curves will be mapped onto each other. This mapping is shown here schematically for an acoustic phonon branch.

We now check whether the transformed functions are equally Bloch functions. We verify that

$$
\begin{aligned}
T\left(\vec{l}_{m}\right) \psi_{P_{a} \vec{k}}(\vec{r}) & =T\left(\vec{l}_{m}\right) \exp \left(i P_{a} \vec{k} \cdot \vec{r}\right) u_{\vec{k}}\left(P_{a}^{-1} \vec{r}\right) \\
& =T\left(\vec{l}_{m}\right) \exp \left(i P_{a} \vec{k} \cdot \vec{r}\right) u_{P_{a} \vec{k}}(\vec{r}) \\
& =\exp \left(i P_{a} \vec{k} \cdot\left(\vec{r}+\vec{l}_{m}\right)\right) u_{\vec{k}}\left(P_{a}^{-1}\left(\vec{r}+\vec{l}_{m}\right)\right) .
\end{aligned}
$$

As $u_{\vec{k}}(\vec{r})$ is a Bloch function it possesses the full lattice periodicity. We, therefore, deduce that

$$
u_{P_{a} \vec{k}}\left(\vec{r}+\vec{l}_{m}\right)=u_{\vec{k}}\left(P_{a}^{-1}\left(\vec{r}+\vec{l}_{m}\right)\right)=u_{\vec{k}}\left(P_{a}^{-1} \vec{r}\right)=u_{P_{a} \vec{k}}(\vec{r}) .
$$

The transformed functions are thus indeed Bloch functions labeled by the transformed wave vector $P_{a} \vec{k}$.

The subspace of the Hilbert space spanned by all $\psi_{P_{a} \vec{k}}(\vec{r})$ is invariant. It has the dimension of the star of the wave vector $\vec{k}$. All functions within this subspace have the same energies. We normally do not speak of essential degeneracies in this case as the respective dispersion sheets do not touch or cross in reciprocal space (see figure 5). Real or essential degeneracies can thus only be obtained, if the star of the wave vector possesses a number of elements that is lower than the order of the point group. This is the case when the group of the wave vector is not trivial, i.e. if it contains at least one element $G_{a} \neq E$.

The point group symmetry introduces an equivalence between certain wave vectors $\vec{k}$. It is thus sufficient to determine the eigenfunctions in a sub-volume of reciprocal space equally denoted as the inequivalent or fundamental volume. As the Brillouin zone reflects by construction ${ }^{9}$ the symmetry of the lattice the fundamental volume is contained within the first Brillouin zone (see figure 8 for an example).

\subsection{Phonon representations for a given wave vector}

The procedure for identifying the irreducible representations of the phonon eigenvectors is fully analogous to the one used for molecular vibrations in section 4.4. In the case of the crystal the role

\footnotetext{
${ }^{9}$ A general point in reciprocal space belongs to the Brillouin zone of a reciprocal lattice vector if it is closest to that reciprocal lattice vector.
} 


\section{EPJ Web of Conferences}

of the molecule is assumed by the primitive cell. The main difference is that contrary to a molecule the primitive cell is not necessarily invariant under symmetry operations. Even simple point group operations may map atoms from the original cell into neighboring primitive cells related by lattice translations. These atoms are necessarily equivalent from a symmetry point of view. We thus may continue to set up equivalence representations $\Gamma_{\text {equiv. }}$. The expression of atomic site representation is, however, no longer adequate. When determining the equivalence representations we have to take into account that atoms in different unit cells are interrelated via translations $\vec{l}(m)$ by a phase factor $\exp i \vec{k} \cdot \vec{l}(m)$. The entries are thus not just 0 and 1 .

The characters $\chi_{\text {equiv. }}$ of the equivalence representation are as usual obtained by summing the entries on the diagonal.

- An atom of the primitive cell that is mapped by an operation of the group of the wave vector onto itself contributes 1 to $\chi_{\text {equiv. }}$.

- Atoms that are transposed to an equivalent position in a different cell contribute a phase factor $\exp (i \vec{k} \cdot \vec{l}(m))$ to $\chi_{\text {equiv. }}$.

- Special attention has to be given to wave vectors $\vec{k}$ on the Brillouin zone boundary. In that case there are operations in the group of the wave vector that involve a translation $\vec{K}(n)$ of the reciprocal lattice. This translation introduces an additional phase factor $\exp (i \vec{K}(n) \cdot \vec{r}(\kappa))$ that now depends on the position $\vec{r}(\kappa)$ of the atom $\kappa$ in the primitive cell.

- The characters of the equivalence transformation are thus given by the general expression

$$
\chi_{\text {equiv. }}\left(\left\{S_{a} \mid \vec{v}\left(S_{a}\right)+\vec{l}(m)\right\}\right)=e^{(i \vec{k} \cdot(\vec{l}+\vec{v}))} \sum_{\kappa=1}^{r} \delta_{\left\{S_{a} \mid \vec{v}\left(S_{a}\right)+\vec{l}(m)\right\} \vec{r}_{\kappa}, \vec{r}_{\kappa}} e^{\left(i \vec{K}(n) \cdot \vec{r}_{\kappa}\right)} .
$$

The equivalence transformation contains all the information of how atoms within the primitive cell transform into each other. By decorating each atom with a displacement vector we get a representation

$$
\Gamma_{\text {phonon }}(\vec{k})=\Gamma_{\text {equiv. }}(\vec{k}) \otimes \Gamma_{\text {vec. }}(\vec{k})
$$

in the vector space of the eigenvectors of the dynamical matrix $\tilde{D}(\vec{k})$. As the group elements of the wave vector are equally symmetry elements of the dynamical matrix the eigenvectors have to transform according to irreducible representations of $\Gamma_{\text {phonon. These irreducible representations can be found with }}$ the standard methods of group theory. ${ }^{10}$

In practice we proceed as follows:

- We consider the vibrational problem for every individual wave vector $\vec{k}$.

- We establish the group of the wave vector $\vec{k}$. For $\vec{k}=0$ the group of the wave vector is identical to the space group of the crystal.

- We establish the characters of the equivalent $\Gamma_{\text {equiv. }}$ and vector $\Gamma_{\text {vec. }}$ representations under the group of the wave vector.

- We determine the irreducible representations of

$$
\Gamma_{\text {phonon }}(\vec{k})=\Gamma_{\text {equiv. }}(\vec{k}) \otimes \Gamma_{\text {vec. }}(\vec{k}) .
$$

- We try to identify the symmetry of the eigenvectors that transform according to these irreducible representations.

Once we have determined the decomposition of $\Gamma_{\text {phonon }}(\vec{k})$ into irreducible representations we can use the projector formalism already encountered in section 2.2 to block diagonalize the dynamical matrix.

\footnotetext{
10 We do not want to go into the intricacies posed by non-symmorphic space groups like multiplier algebras. These can be found in dedicated text books and review articles [1, 3].
} 


\section{Contribution of Symmetries in Condensed Matter}

Let us denote the multiplicity and the dimension of a specific irreducible representation $\mathrm{IR}_{j}$ by $m_{j}$ and $s_{j}$, respectively. In that case $m_{j} \cdot s_{j}$ eigenvectors will transform according to $\mathrm{IR}_{j}$, with $\sum_{j} m_{j} \cdot s_{j}=3 r$. The diagonal matrix will thus diagonalize into blocks of dimension $m_{j} \times m_{j}$. If $\mathrm{IR}_{j}$ has a dimension $s_{j}>1$ then there will be $s_{j}$ blocks of that form in the decomposition. Mathematically speaking we can find an orthogonal matrix $\tilde{X}$ of dimension $3 N \times 3 N$ such that

$$
\tilde{D}^{X}=\tilde{X}^{-1} \tilde{D} \tilde{X}=\left(\begin{array}{ccc}
\left(\begin{array}{ccc}
a_{1,1} & \ldots & a_{1, m_{1}} \\
\ldots & \ldots & \ldots \\
a_{m_{1}, 1} & \ldots & a_{m_{1}, m_{1}}
\end{array}\right) \\
\\
0
\end{array}\right.
$$

\subsection{Symmetry coordinates}

If we apply the transformation $\tilde{X}$ to the $3 N$ cartesian components of the atomic displacements in the unit cell then we obtain the so-called symmetry coordinates. These are vectors that transform according to the irreducible transformations $\mathrm{IR}_{j}$. By definition a symmetry coordinate can only possess components that are interrelated by symmetry operations. In other words, symmetry coordinates are fully symmetry adapted. They thus do e.g. not mix cartesian components of non-equivalent atoms and in particular of different atom types. There will be $m_{j} \cdot s_{j}$ symmetry coordinates for every representation. For $s_{j}>1$ subsets of symmetry coordinates for a given irreducible transformation would transform into each other. As the projection formalism can be fully programmed there exist computer codes [5, 15], e.g. incorporated in the program package UNISOFT [16], that automatically determine the symmetry coordinates as well as the block-diagonal form of the dynamical matrix.

\subsection{Example: Phonons in $\mathrm{NaCl}$}

We want to illustrate the general procedure with a simple example. Following [1] we choose the rock salt NaCl-structure. This structure is at the same time simple and highly symmetric. As it features two atoms per primitive cell $\mathrm{NaCl}$ possesses optic and acoustic branches.

The space group is \#225( $F m 3 m) O_{h}^{5}$ (see figure 6). We are dealing with two interpenetrating FCC lattices of $\mathrm{Na}$ and $\mathrm{Cl}$ ions. The primitive cell of a FCC lattice is rhombohedral. It contains one $\mathrm{Na}$ and one $\mathrm{Cl}$ ion, respectively. As $\mathrm{Na}$ and $\mathrm{Cl}$ ions are not equivalent we immediately deduce that the equivalence representation is the trivial representation. All the symmetry elements will necessarily map "the" $\mathrm{Na}$ and "the" $\mathrm{Cl}$ ion in the primitive cell on "the" $\mathrm{Na}$ and "the" $\mathrm{Cl}$ ion in another primitive cell.

We will first deal with the wave vector $\vec{k}=0$. In this case the group of the wave vector coincides with the space group of the crystal. The star of $\vec{k}=0$ is, therefore, identical with $\vec{k}=0$ itself. All the phase factors that could appear in expression 5.8 are zero. The equivalence representation is thus identical to a product of the $\Gamma_{1}$ representation of the point group $O_{h}$ with itself (see Table 5)

$$
\Gamma_{\text {equiv. }}(\vec{k}=0)=2 \Gamma_{1} \text {. }
$$

A vector transforms under the group $O_{h}$ as $\Gamma_{15}$. The basis function of $\Gamma_{15}$ is the vector $(x, y, z)$ (see Table 5). The $\vec{k}=0=$ ( $\Gamma$-point) phonons transform, therefore, according to

$$
\Gamma_{\text {phonon }}(\Gamma)=\Gamma_{\text {vec. }}(\Gamma) \otimes \Gamma_{\text {equiv. }}(\Gamma)=2 \Gamma_{1} \otimes \Gamma_{15}=2 \Gamma_{15}
$$



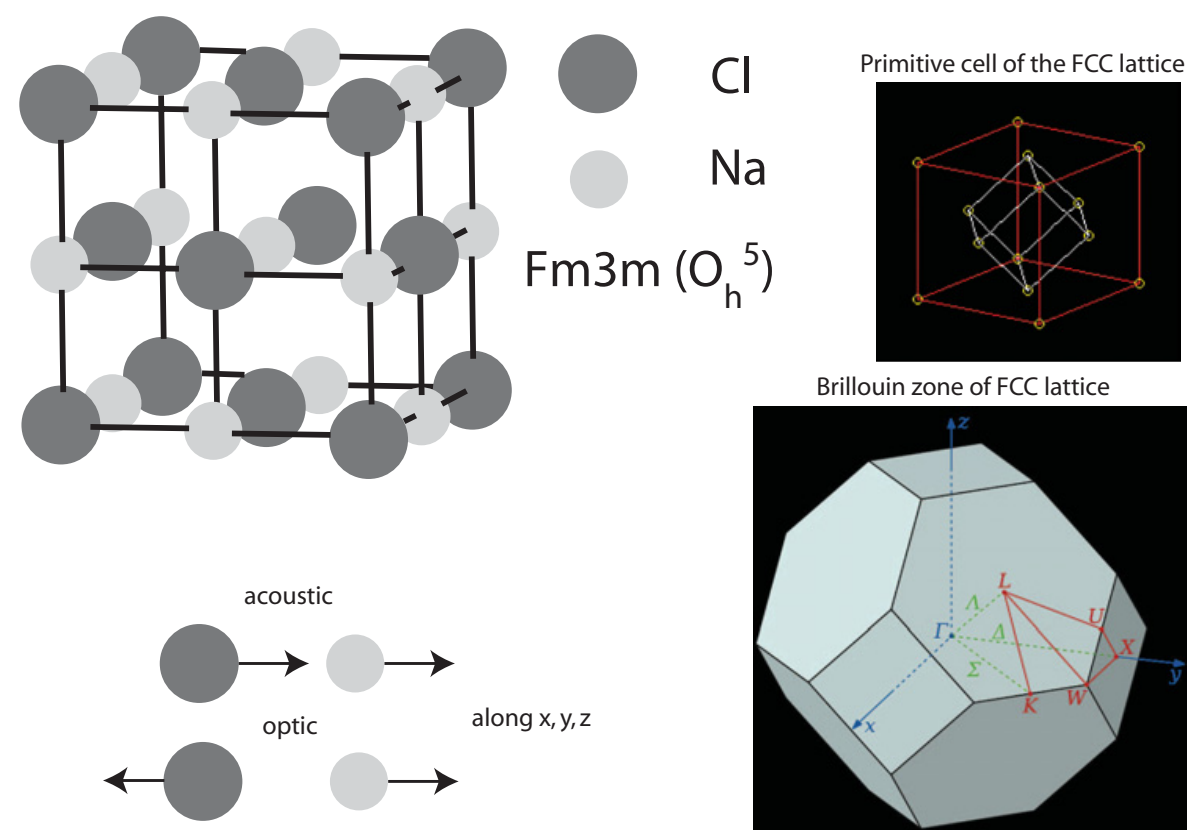

Brillouin zone of FCC lattice

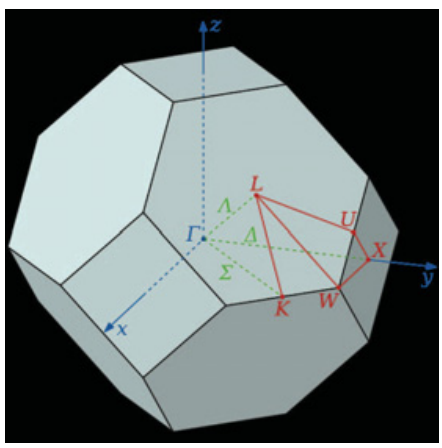

Figure 6. Structure of $\mathrm{NaCl}$. $\mathrm{Na}$ and $\mathrm{Cl}$ ions occupy positions of $\mathrm{FCC}$ lattices, one displaced with respect to the other by half a lattice vector. The space group symmetry is \#225( $F m 3 m) O_{h}^{5}$. The primitive cell is rhombohedral.

Table 5. Character table for a selected set of irreducible representations of the group $O_{h}$.

\begin{tabular}{|l|l||r|r|r|r|r|r|r|r|r|r|}
\hline$\Gamma$ & basis function & $E$ & $3 C_{4}^{2}$ & $6 C_{4}$ & $6 C_{2}$ & $8 C_{3}$ & $i$ & $3 i C_{4}^{2}$ & $6 i C_{4}$ & $6 i C_{2}$ & $8 i C_{3}$ \\
\hline$\Gamma_{1}$ & 1 & 1 & 1 & 1 & 1 & 1 & 1 & 1 & 1 & 1 & 1 \\
$\Gamma_{15}$ & $x, y, z$ & 3 & -1 & 1 & -1 & 0 & -3 & 1 & -1 & 1 & 0 \\
\hline
\end{tabular}

The dynamical matrix thus diagonalizes into three blocks of dimension $(2 \times 2)$

$$
\tilde{D}^{X}=\left(\begin{array}{ccc}
\left(\begin{array}{cc}
a_{1,1} & a_{1,2} \\
a_{2,1} & a_{2,2}
\end{array}\right) & 0 & 0 \\
0 & \left(\begin{array}{cc}
b_{1,1} & b_{1,2} \\
b_{2,1} & b_{2,2}
\end{array}\right) & 0 \\
0 & 0 & \left(\begin{array}{ll}
c_{1,1} & c_{1,2} \\
c_{2,1} & c_{2,2}
\end{array}\right)
\end{array}\right) .
$$

Due to the cubic symmetry the symmetry coordinates are in this case very simple. They can be chosen to correspond to the cartesian coordinates of the $\mathrm{Na}$ and $\mathrm{Cl}$ ion, respectively. In other words, the cartesian coordinate system is the symmetry adapted coordinate system for cubic symmetry, which is not surprising. The $(2 \times 2)$-dimensional blocks mix the $x-, y-, z$-coordinates of the $\mathrm{Na}$ and $\mathrm{Cl}$ ion, respectively.

Via the decomposition we arrive at two sets of eigenfunctions each three-times degenerate. We know that one set has to correspond to the acoustic modes, while the other set contains the optic modes. Due to the cubic symmetry we can a priori choose the eigenvectors along arbitrary orthogonal directions. The conventional choice corresponds to displacements along the three cubic lattice directions $\vec{a}_{i}$. As symmetry does not relate atoms of different atom types it will not restrict the phases of the two sublattices. To fix these phase we need physical arguments. The acoustic modes correspond for $\vec{k}$ to a free translation of the whole crystal. All atoms thus move in phase and the frequencies extrapolate towards 


\section{Contribution of Symmetries in Condensed Matter}

Table 6. Character table of the group $C_{4 v}$ for the group of the wave vector at a $\Delta$-point $(\Delta=2 \pi / a(x, 0,0))$. Only the representations relevant for expressing a vector are given. We equally give the characters for the $\Gamma$-point representation $\Gamma_{15}$ in that group.

\begin{tabular}{|l|l||r|r|r|r|r|}
\hline$\Gamma$ & basis function & $\mathrm{E}$ & $C_{4}^{2}$ & $2 C_{4}$ & $2 i C_{4}^{2}$ & $2 i C_{2}^{\prime}$ \\
\hline$\Delta_{1}$ & $x$ & 1 & 1 & 1 & 1 & 1 \\
$\Delta_{5}$ & $y, z$ & 2 & -2 & 0 & 0 & 0 \\
\hline & \multicolumn{7}{|c|}{} \\
\hline$\Gamma_{15}$ & \multicolumn{1}{|c|}{3} & -1 & 1 & 1 & 1 \\
\hline
\end{tabular}

Table 7. Character table of the group $C_{4 v}$ for the group of the wave vector at the $X$-point. Only the representations relevant for a vector are given.

\begin{tabular}{|l|l||r|r|r|r|r|r|r|r|r|r|}
\hline$\Gamma$ & basis function & $\mathrm{E}$ & $2 C_{4 \perp}^{2}$ & $C_{4 \|}^{2}$ & $2 C_{4 \|}^{2}$ & $2 C_{2}$ & $\mathrm{i}$ & $2 i C_{4 \perp}^{2}$ & $i C_{4 \|}^{2}$ & $2 i C_{4 \| \mid}$ & $2 i C_{2}$ \\
\hline$X_{4}^{\prime}$ & $x$ & 1 & -1 & 1 & 1 & -1 & -1 & 1 & -1 & -1 & 1 \\
$X_{5}^{\prime}$ & $y, z$ & 2 & 0 & -2 & 0 & 0 & -2 & 0 & 2 & 0 & 0 \\
\hline
\end{tabular}

zero. Eigenvectors in one set have to be orthogonal to eigenvectors in the other set. The optic modes thus correspond to out-of-phase motion of the two sub-lattices (see figure 6).

When we leave the zone center along one of the main reciprocal lattice directions then the symmetry of the wave vector $\vec{k}$ reduces to $C_{4 v}$. The character table for the wave vector $\vec{k}$ is given in Table 6 . We realize that a vector now transforms according to

$$
\Gamma_{\text {vec. }}(\Delta)=\Delta_{1}+\Delta_{5}
$$

This gives for the phonon representation

$$
\Gamma_{\text {phonon }}(\Delta)=\Gamma_{\text {vec. }}(\Delta) \otimes \Gamma_{\text {equiv. }}(\Delta)=2 \Delta_{1} \otimes\left(\Delta_{1}+\Delta_{5}\right)=2 \Delta_{1}+2 \Delta_{5} .
$$

For every symmetry type (irreducible representation) we obtain again 2 sets of eigenvectors. As these modes have to connect with those at the $\Gamma$-point we can immediately deduce that the three-fold degenerate acoustic and optic branches split into a single and two doubly degenerate branches each. Inspection of the basis functions in Table 6 tells us that the $\Delta_{1}$ mode transforms like the component of a vector along the $\Delta$-direction. The $\Delta_{1}$-modes have thus a purely longitudinal character. The $\Delta_{5}$ modes transform like the components of a vector perpendicular to the $\Delta$-direction. We are thus dealing with transverse modes. As due to the 4-fold symmetry axis the two components are equivalent we get two degenerate transverse branches.

The $\Gamma_{15}$ representation at the $\Gamma$-point thus decomposes into one $\Delta_{1}$ plus one $\Delta_{5}$ representation. We could have deduced this directly by inspecting the characters of $\Gamma_{15}$ under the operations of $C_{2 v}$. Under $C_{2 v}$ the representation $\Gamma_{15}$ becomes reducible and the reduction is equal to $\Delta_{1}+\Delta_{5}$. Such compatibility relations can be obtained for all points in reciprocal space that feature a change in the point group of the wave vectors. They are for example tabulated in [13, 14].

When we continue to the $X$-point the group of the wave vector changes again. With the help of Table 7 the representation of a vector in the new group is identified as

$$
\Gamma_{\text {vec. }}(X)=X_{4}^{\prime}+X_{5}^{\prime} \text {. }
$$

This leads to the phonon representation

$$
\Gamma_{\text {phonon }}(X)=\Gamma_{\text {vec. }}(X) \otimes \Gamma_{\text {equiv. }}(X)=2 X_{4}^{\prime}+2 X_{5}^{\prime} .
$$

From the inspection of the basis vectors we get immediately the compatibility relation with the $\Delta$ symmetries

$$
\Gamma_{15} \rightarrow \Delta_{1}+\Delta_{5} \rightarrow X_{4}^{\prime}+X_{5}^{\prime}
$$

Measured and calculated dispersion relations for $\mathrm{NaCl}$ are shown in figure 7 . 


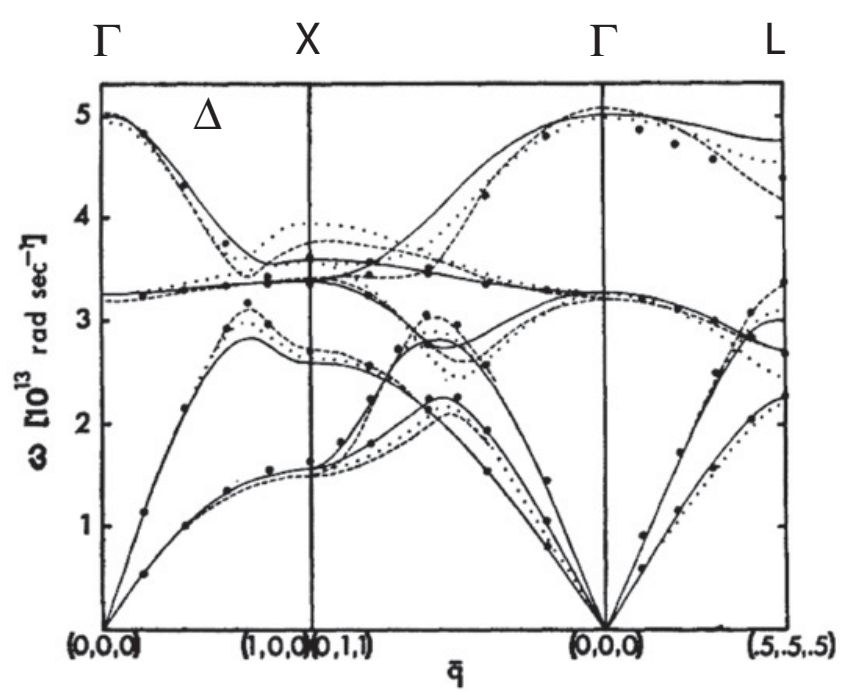

Figure 7. Phonon dispersion modes in $\mathrm{NaCl}$. The points have been measured with inelastic neutron scattering. The lines correspond to different lattice dynamical models. The symbols for special points are defined in figure 6 . Details are given in [17].

\subsection{Example: phonons in quartz}

We now will very qualitatively discuss the phonons in a more complex structure. This will in particular allow us to highlight a few aspects related to non-symmorphic space groups. We choose the example of quartz. Quartz exists in a low-temperature $\alpha$ and a high-temperature $\beta$-phase. The primitive cell of quartz is hexagonal. The primitive lattice vectors are given in cartesian coordinates by

$$
\vec{a}=a \vec{e}_{1}, \quad \vec{b}=-\frac{1}{2} a \vec{e}_{1}+\frac{\sqrt{3}}{2} a \vec{e}_{2}, \quad \vec{c}=c \vec{e}_{3}
$$

with lattice constants of $a \approx 4.91 \AA$ and $c \approx 5.41 \AA$, respectively.

The reciprocal lattice is again hexagonal. The reciprocal lattice vectors are given by

$$
\vec{a}^{*}=\frac{2 \pi}{a}\left(\vec{e}_{1}+\frac{1}{\sqrt{3}} \vec{e}_{2}\right), \quad \vec{b}^{*}=\frac{4 \pi}{a \sqrt{3}} \vec{e}_{2}, \quad \vec{b}^{*}=\frac{2 \pi}{c} \vec{e}_{3} .
$$

The basis within the primitive cell is composed of three chemical $\mathrm{SiO}_{2}$ units. These 9 atoms with their 27 degrees of freedom give 27 phonon branches.

$\alpha$-quartz features two symmetry axes. A three-fold screw-axis along $\vec{c}$ and a two-fold rotation axis along $\vec{a}$. Depending on the sense of rotation of the screw-axis we get a different chirality. The chirality has, however, no particular influence on the dispersion relations. Going from the $\alpha$ to the $\beta$-phase the three-fold symmetry becomes a six-fold symmetry.

The positions of the atoms in the primitive cell can be generated with the help of the symmetry operators from 6 structural parameters. Apart from the lattice constants we need a representative silicon and oxygen ion. These are given by

$$
\vec{r}_{\mathrm{Si}}=u \vec{a} \quad \vec{r}_{\mathrm{O}}=x \vec{a}+y \vec{b}-z \vec{c}
$$

with

$$
x \approx 0.414, \quad y \approx 0.266, \quad z \approx 0.119, \quad u \approx 0.470 .
$$




\section{Contribution of Symmetries in Condensed Matter}

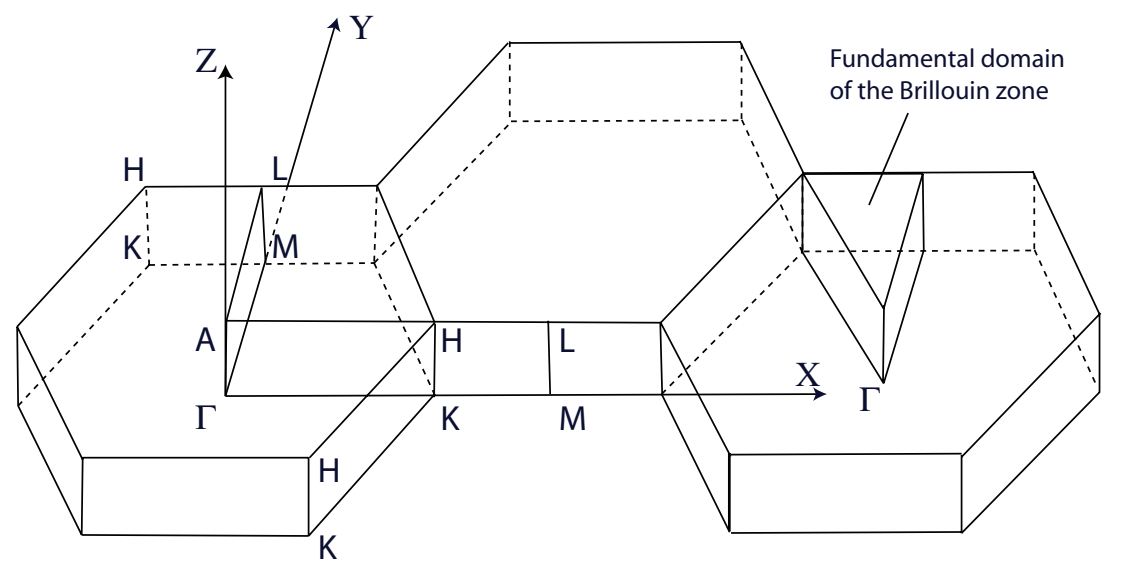

Figure 8. Reciprocal lattice of quartz. High-symmetry points are labelled by special symbols. All the information concerning the excitations is contained in the fundamental domain of a Brillouin zone. The fundamental volume makes up only $1 / 24$ of the total zone.

$\beta$-quartz possesses a higher symmetry reducing the number of free parameters to 2 :

$$
x, \quad y=x / 2, \quad z=1 / 6, \quad u=1 / 2 .
$$

The phonon spectra of $\alpha$-quartz are shown in figure 9. In the $\Gamma-A$-direction the dynamical matrix decomposes into three irreducible representations denoted $\Delta_{1}, \Delta_{2}$ and $\Delta_{3}$. They are all one-dimensional. Each representation features one acoustic mode. The compatibility relations show that $\Delta_{2}$ et $\Delta_{3}$ merge into two-dimensional representations at the $\Gamma$-point. They thus become degenerate in pairs. The same holds for the $\Delta_{1}$ and $\Delta_{2}$ representations at the zone boundary (A-point). These degeneracies imposed by symmetry allow us to unfold the dispersion curves. We thus can represent them in the way we would do for a crystal that had a three times smaller primitive cell and thus a three times larger Brillouin zone along this direction. The unfolding is in no way a purely graphical exercise. In a neutron scattering experiment one would e.g. observe a real continuity of intensity among curves that join at the zone center and at the zone boundary. If we perform the measurement along the hexagonal axis we never observe more than 9 branches simultaneously. These 9 branches would always belong to only one of the three representations. This is an example of selection rules that can be derived purely from symmetry considerations. Details can e.g. be found in [20].

\subsection{Conservation properties beyond the harmonic approximation}

To see how symmetry can help us beyond the harmonic approximation we consider conservation laws. It is a fundamental theorem of quantum mechanics that any continuous symmetry is accompanied by a conserved quantity. A well-know example is the conservation of momentum of a free particle. As there are no external reference points, like e.g. springs that would attach the particle to walls, the Hamiltonian is invariant under a translation

$$
\vec{r} \rightarrow \vec{r}+\vec{r}_{0} .
$$

The translational symmetry thus states the fact that all reference systems are equivalent for a free particle. The action of a translation on the wave function of a free particle consists in a phase change of $\vec{k} \cdot \vec{r}_{0}$. It is, therefore, described by the quantum mechanical operator

$$
T_{\vec{r}_{0}}=\exp \left(\frac{i}{\hbar} \vec{p} \cdot \vec{r}_{0}\right) \text {. }
$$



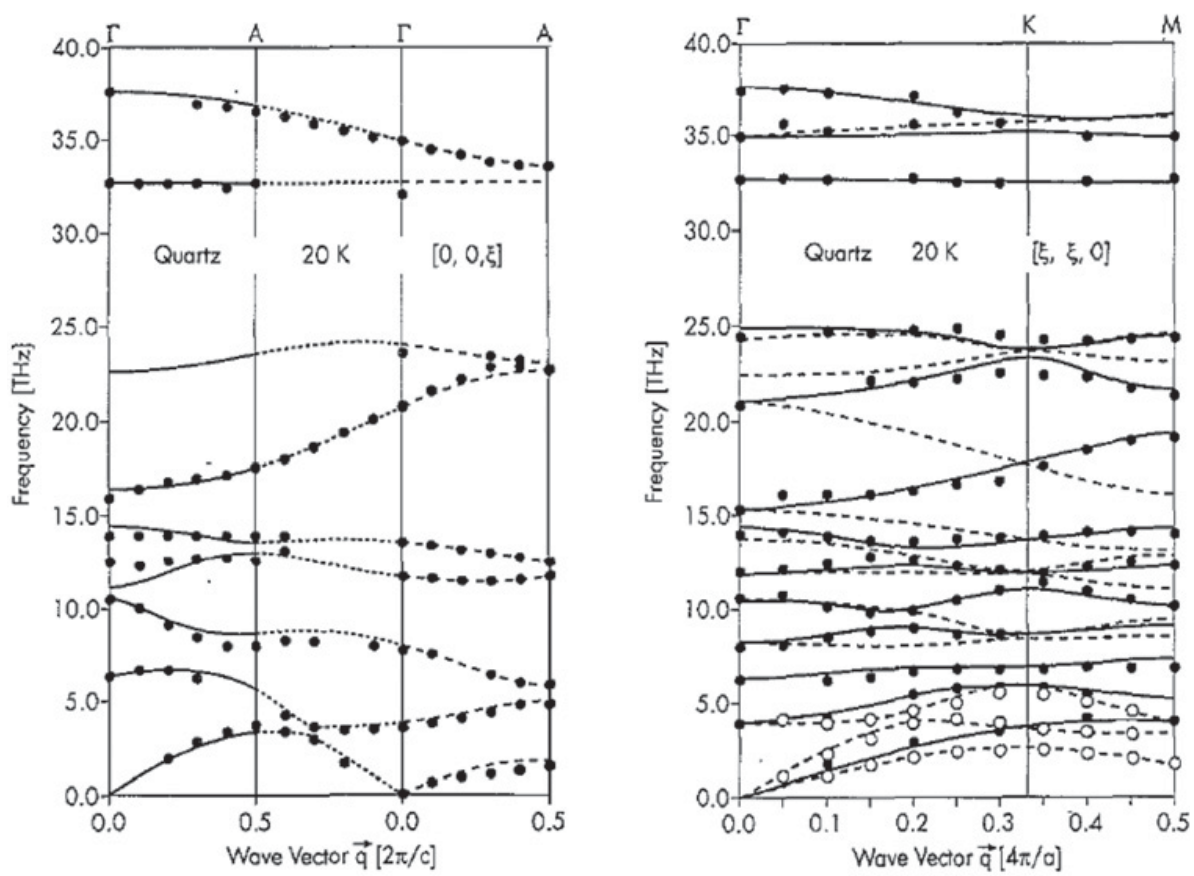

Figure 9. Dispersion curves for $\alpha$-quartz along the two high-symmetry directions. Circles correspond to experimental measurements obtained with inelastic neutron scattering [18]. The lines give the result of lattice dynamical model calculations [19].

$T_{\vec{r}_{0}}$ commutes evidently with

$$
H=p^{2} / 2 m
$$

and its eigenvalues are thus constants of motion, i.e. they do not change with time. In other terms, if the particle is in an eigenstate of $T_{\vec{r}_{0}}$ then it will remain there forever.

If we are dealing with interacting particles we can still freely translate the reference frame without changing the Hamiltonian provided there are no external fields. It can be shown that the wave function then acquires the phase $\sum_{i} \vec{k}_{i} \cdot \vec{r}_{0}$, i.e. the translation is described by the operator

$$
T_{\vec{r}_{0}}=\exp \left(\frac{i}{\hbar} \sum_{i} \vec{p}_{i} \cdot \vec{r}_{0}\right)
$$

The fact that $\left[T_{\vec{r}_{0}}, H\right]=0$ leads to the conservation of total momentum. This conservation will naturally persist in a crystal and the existence of three zero-frequency modes bears witness to it.

That the total moment of a body is conserved is a trivial and in practice not very useful result. In the case of a crystal we can, however, go a step further. The existence of periodicity implies that we can perform concerted permutations of the operators pertaining to the ions without changing the physics. ${ }^{11}$ Let us reconsider the Hamiltonian of the solid expressed in eq. (2.2) and reproduced below:

$$
H=\sum_{i} \frac{\vec{p}_{i}^{2}}{2 m_{i}}+\sum_{I} \frac{\vec{p}_{I}^{2}}{2 M_{I}}+\sum_{i \leq j} \frac{e^{2}}{\left|\vec{r}_{i}-\vec{r}_{j}\right|}+\sum_{I \leq J} \frac{Z_{I} Z_{J} e^{2}}{\left|\vec{R}_{I}-\vec{R}_{J}\right|}-\sum_{i, I} \frac{Z_{I} e^{2}}{\left|\vec{r}_{i}-\vec{R}_{I}\right|} .
$$

\footnotetext{
11 We follow here the argumentation of Ashcroft and Mermin [7].
} 


\section{Contribution of Symmetries in Condensed Matter}

Using the adiabatic approximation we describe the interactions involving the ions via a potential

$$
\frac{1}{2} \sum_{I, J} \Phi\left(\vec{R}_{I}^{0}+\vec{u}\left(\vec{R}_{I}^{0}\right)-\vec{R}_{J}^{0}-\vec{u}\left(\vec{R}_{J}^{0}\right)\right)
$$

that depends only on the relative distances between the ions. ${ }^{12}$ The Hamiltonian for the ions then simplifies to

$$
H=\sum_{I} \frac{\vec{p}^{2}\left(\vec{R}_{I}^{0}\right)}{2 M_{I}}+\frac{1}{2} \sum_{I, J} \Phi\left(\vec{R}_{I}^{0}+\vec{u}\left(\vec{R}_{I}^{0}\right)-\vec{R}_{J}^{0}-\vec{u}\left(\vec{R}_{J}^{0}\right)\right) .
$$

By employing the notation $\vec{p}\left(\vec{R}_{I}^{0}\right)$ we stress the fact that the momentum operators are the conjugate operators to the ion coordinates at the various sites. To stay as general as possible we may add an arbitrary number of particles to the system. These particles are supposed to interact among each other and with the ions. Possible candidates for such particles are the Bloch electrons or external probe particles like neutrons. We thus end up with the Hamiltonian

$$
\begin{aligned}
H= & \sum_{I} \frac{\vec{p}^{2}\left(\vec{R}_{I}^{0}\right)}{2 M_{I}}+\frac{1}{2} \sum_{I, J} \Phi\left(\vec{R}_{I}^{0}+\vec{u}\left(\vec{R}_{I}^{0}\right)-\vec{R}_{J}^{0}-\vec{u}\left(\vec{R}_{J}^{0}\right)\right) \\
& +\sum_{i} \frac{\vec{p}^{2}\left(\vec{r}_{i}\right)}{2 m_{i}}+\frac{1}{2} \sum_{i, j} v_{i, j}\left(\vec{r}_{i}-\vec{r}_{j}\right)+\sum_{i, I} w_{i}\left(\vec{r}_{i}-\vec{R}_{I}^{0}-\vec{u}\left(\vec{R}_{I}^{0}\right)\right) .
\end{aligned}
$$

We may now translate the whole system by an arbitrary vector $\vec{\tau}$. This shifts the coordinates like

$$
\begin{aligned}
& \vec{r}_{i} \rightarrow \vec{r}_{i}+\vec{\tau} \\
& \vec{u}\left(\vec{R}_{I}^{0}\right) \rightarrow \vec{u}\left(\vec{R}_{I}^{0}\right)+\vec{\tau}
\end{aligned}
$$

and is related to a conservation of the total momentum that we have already talked about. If now $\tau$ corresponds to a reciprocal lattice vector $\vec{l}$ then the translation can be described differently within the ionic subsystem, i.e. by a concerted permutation of the particles. To get the same result we just have to replace all the ions by those ions that are found at a distance that corresponds to the lattice vector $\vec{l}$. Formally we substitute

$$
\begin{aligned}
& \vec{r}_{i}^{0} \rightarrow \vec{r}_{i}^{0}+\vec{l} \\
& \vec{R}_{I}^{0} \rightarrow \vec{R}_{I}^{0}-\vec{l}=\vec{R}_{I^{\prime}}^{0} \\
& \vec{u}\left(\vec{R}_{I}^{0}\right) \rightarrow \vec{u}\left(\vec{R}_{I}^{0}-\vec{l}\right)=\vec{u}\left(\vec{R}_{I^{\prime}}^{0}\right) \\
& \vec{P}(\vec{R}) \rightarrow \vec{P}\left(\vec{R}_{I}^{0}-\vec{l}\right)=\vec{P}\left(\vec{R}_{I^{\prime}}^{0}\right) .
\end{aligned}
$$

Both procedures (eq. (5.28) and eq. (5.29)) are absolutely equivalent for a lattice translation in an isolated periodic system. The differences become evident as soon as we destroy either of the two requirements.

- If we break isolation but preserve periodicity by coupling every primitive cell of the crystal in an identical way to an external field ${ }^{13}$ then the Hamiltonian will still be invariant with respect to the permutation of identical particles. It will, however, no longer be invariant with respect to translations. This implies that the momentum will not be conserved. The permutation is thus the more fundamental symmetry of a periodic system.

\footnotetext{
12 For simplicity we choose pair potentials. The results will, however be valid for any kind of inter-ionic potential.

13 In the simple example given in [7] every ion is attached via a spring to a wall.
} 


\section{EPJ Web of Conferences}

- If we preserve the isolation but break periodicity for e.g. by introducing mass defects then it is the other way round. We still can translate the system as a whole preserving overall momentum but we can no longer permute the ions without changing the Hamiltonian. The translation symmetry is thus the more fundamental symmetry for an isolated system.

To exploit the permutation symmetry we have to identify the quantum mechanical operator that goes along with it. We are thus looking for an unitary operator of the general form

$$
U_{\text {perm. }}(\vec{l})=\exp (i \mathbf{K}(\vec{l}))
$$

that assures for every lattice vector $\vec{l}$ that

$$
\begin{aligned}
\exp (i \mathbf{K}(\vec{l})) \vec{u}\left(\vec{R}_{I}^{0}\right) \exp (-i \mathbf{K}(\vec{l})) & =\vec{u}\left(\vec{R}_{I}^{0}-\vec{l}\right) \\
\exp (i \mathbf{K}(\vec{l})) \vec{P}\left(\vec{R}_{I}^{0}\right) \exp (-i \mathbf{K}(\vec{l})) & =\vec{P}\left(\vec{R}_{I}^{0}-\vec{l}\right)
\end{aligned}
$$

It can be shown that $\exp (i \mathbf{K}(\vec{l}))$ fulfills this task if we choose $\mathbf{K}(\vec{l})$ such that

$$
\mathbf{K}(\vec{l})\left|\left\{n_{j}(\vec{k})\right\}>=\sum_{\vec{k}} \sum_{j} n_{j}(\vec{k})(\vec{k} \cdot \vec{l})\right|\left\{n_{j}(\vec{k})\right\}>,
$$

with $\mid\left\{n_{j}(\vec{k})\right\}>$ denoting the eigenfunctions of the system of coupled harmonic oscillators in the harmonic approximation. The sum runs over all wave vectors $\vec{k}$ in the first Brillouin zone and over all phonon branches $j$. The number $n_{j}(\vec{k})$ gives the occupation number of a given oscillator and will depend on temperature. $\mathbf{K}(\vec{l})$ is called the operator of crystal momentum. It extracts from a quantum state represented in the basis of the phonon wave functions the sum of the projections of the wave vectors $\vec{k}$ onto the lattice vector $\vec{l}$.

We do not want to give the proof of eqs. (5.30) and (5.31) as it does not add insight into the matter of symmetry. ${ }^{14}$ We want to stress thought, that formulating $\mathbf{K}(\vec{l})$ in the basis of phonon wave functions does not mean that this operator is only useful in the harmonic approximation. Quite to the contrary, as we will show now the operator $\mathbf{K}(\vec{l})$ demonstrates its full strength only when applied to anharmonic systems.

If wee ignore for the moment the possible presence of additional particles then the operators $U_{\text {perm. }}(\vec{l})$ commute with the ionic Hamiltonian and thus constitute constants of motion. Therefore, if the system is in an eigenstate of $U_{\text {perm. }}(\vec{l})$ then it will stay in that eigenstate forever. Let us prepare an eigenstate of the system $\mid \psi>$ that at a given moment in time corresponds to a particular set of phonons $\left\{n_{j}(\vec{k})\right\}$. Then $\mid \psi>$ will be an eigenfunction of the operators $U_{\text {perm. }}(\vec{l})$ as

$$
U_{\text {perm. }}(\vec{l})\left|\left\{n_{j}(\vec{k})\right\}>=\exp \left(\sum_{\vec{k}} \sum_{j} n_{j}(\vec{k})(\vec{k} \cdot \vec{l})\right)\right|\left\{n_{j}(\vec{k})\right\}>\quad \forall \vec{l} .
$$

As the $U_{\text {perm. }}(\vec{l})$ commute with the Hamitonian the $\psi$ will remain an eigenfuction of the $U_{\text {perm. }}(\vec{l})$. Due to the anharmonic interactions the occupation numbers describing $\psi$ in terms of phonons will, however, evolve with time. Let us denote these modified occupation numbers by $\left\{n_{j}^{\prime}(\vec{k})\right\}$. Then we require that the new set fulfills the conditions

$$
\exp \left(\sum_{\vec{k}} \sum_{j} n_{j}(\vec{k})(\vec{k} \cdot \vec{l})\right)=\exp \left(\sum_{\vec{k}} \sum_{j} n_{j}^{\prime}(\vec{k})(\vec{k} \cdot \vec{l})\right) \quad \forall \vec{l} .
$$

\footnotetext{
14 The reader is referred to [7].
} 
a)

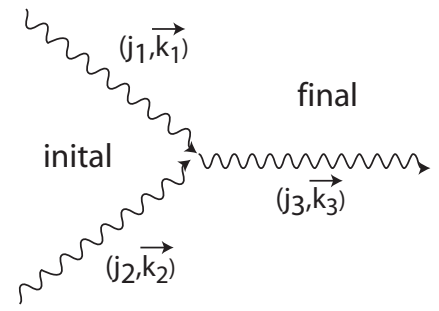

b)

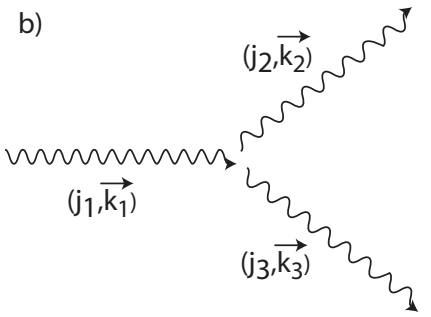

c)

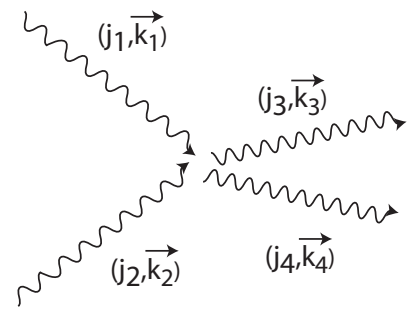

d)

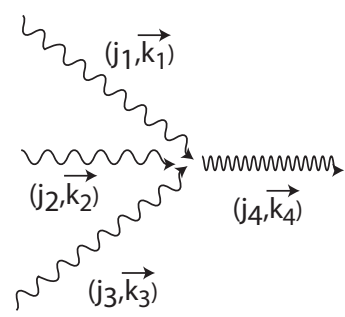

e)

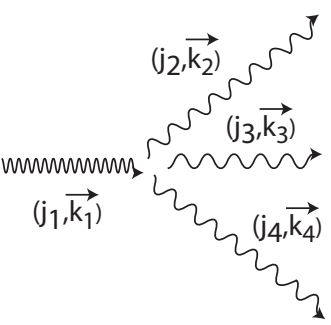

Figure 10. Schematic description of anharmonic processes. Phonons can either decay or merge. In all cases energy and crystal momentum have to be conserved.

We can reformulate these requirements as

$$
\exp \left(\left[\sum_{\vec{k}} \sum_{j} n_{j}(\vec{k}) \vec{k}-n_{j}^{\prime}(\vec{k}) \vec{k}\right] \cdot \vec{l}\right)=1 \quad \forall \vec{l} .
$$

They can only be simultaneously satisfied if the expression in square brackets corresponds to a vector of the reciprocal lattice

$$
\left[\sum_{\vec{k}} \sum_{j} n_{j}(\vec{k}) \vec{k}-n_{j}^{\prime}(\vec{k}) \vec{k}\right]=\vec{K}
$$

The crystal momentum is thus preserved up to an arbitrary reciprocal lattice vector $\vec{K}$. The crystal momentum is never to be confounded with the normal momentum connected with particle motion. This becomes most evident in the case of transverse phonons. For transverse modes the ions vibrate perpendicular to the wave vector $\vec{k}$ while the crystal momentum is directed along $\vec{k}$.

Anharmonic processes are described in a perturbative approach by the decay and recombination of phonons. Such processes are shown in figure 10. Together with the conservation of energy the conservation of crystal momentum places severe constraints on such processes. There is an important distinction between normal and so-called Umklapp processes (see figure 11). The normal processes do not redirect the crystal momentum. It can be shown that they, therefore, do not contribute to thermal resistance.

We now add an additional particle to the system of ions. This could be a Bloch electron as discussed in section 3 or an external probe particle like a neutron. The presence of such particles is supposed not to perturb the periodicity of the crystal lattice. If translated by a reciprocal lattice vector $\vec{l}$ the Bloch electron or neutron will encounter exactly the same interactions only with different ions. The translation 


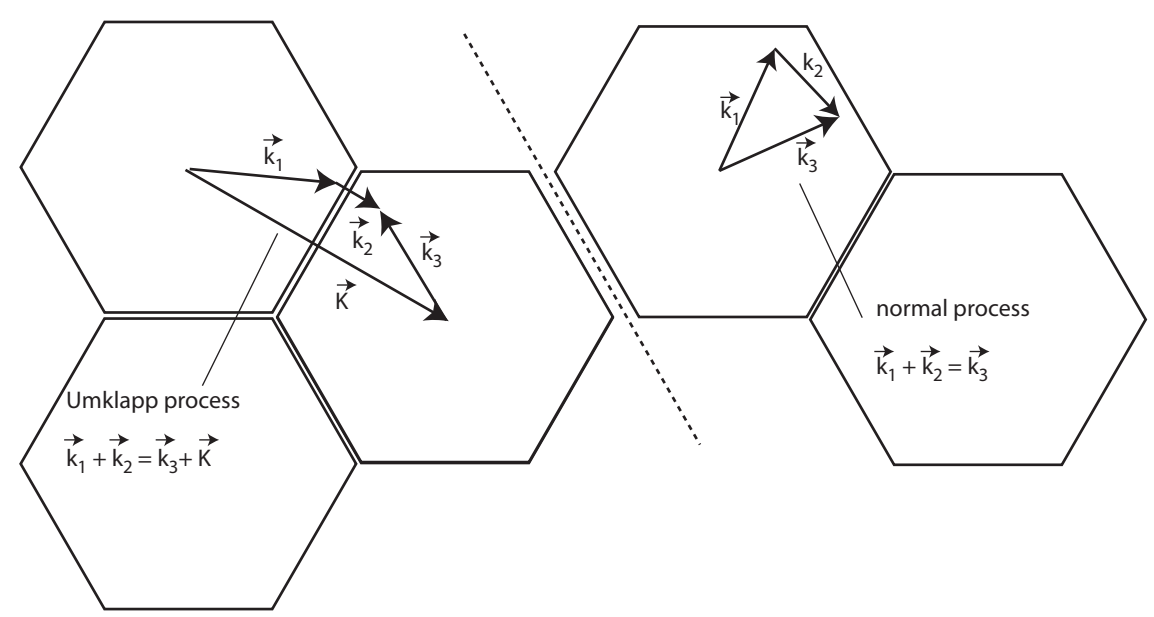

Figure 11. Umklapp and normal phonon processes. For normal processes the crystal momentum is conserved in absolute terms. For Umklapp processes the crystal momentum changes by a reciprocal lattice vector $\vec{K}$ (see eq. (5.36)).

operator of the particle is given by expression (5.23)

$$
T_{\text {part. }}(\vec{l})=\exp \left(\frac{i}{\hbar} \vec{p} \cdot \vec{l}\right) .
$$

The invariance of the total particle-ion Hamiltonian under the transformation expressed in (5.29) implies that the product

$$
T_{\text {part. }}(\vec{l}) \cdot U_{\text {perm. }}(\vec{l})
$$

commutes with the Hamiltonian. If in the beginning the crystal is in an eigenstate of the harmonic system and the particle in an eigenstate of the momentum operator with $\vec{p}=\hbar \vec{q}_{\text {ini. }}$. the combined system is in an eigenstate of

$$
T_{\text {part. }}(\vec{l}) U_{\text {perm. }}(\vec{l}) \quad \forall \vec{l}
$$

with eigenvectors

$$
\exp \left(\left[\vec{q}_{\text {ini. }}+\sum_{\vec{k}} \sum_{j} n_{j}(\vec{k}) \vec{k}\right] \cdot \vec{l}\right) .
$$

Due to the interactions including anharmonicities neither the particle nor the ionic system will remain in their initial state. Using the same arguments that we have deployed to deduce expression (5.36) we can show that the change in crystal momentum is related to the change in particle momentum up to a reciprocal lattice vector. Formally

$$
\left[\sum_{\vec{k}} \sum_{i} n_{i}(\vec{k}) \vec{k}-n_{i}^{\prime}(\vec{k}) \vec{k}\right]+\vec{K}=\vec{q}_{\text {final }}-\vec{q}_{\text {ini. }} .
$$

This is a very general statement of great importance for all kinds of scattering problems. We can for example immediately deduce that the change in momentum of a neutron scattered by phonons corresponds to the change in crystal momentum of the phonon system before and after the scattering process, up to a reciprocal lattice vector. This statement holds independent of the number of phonons 


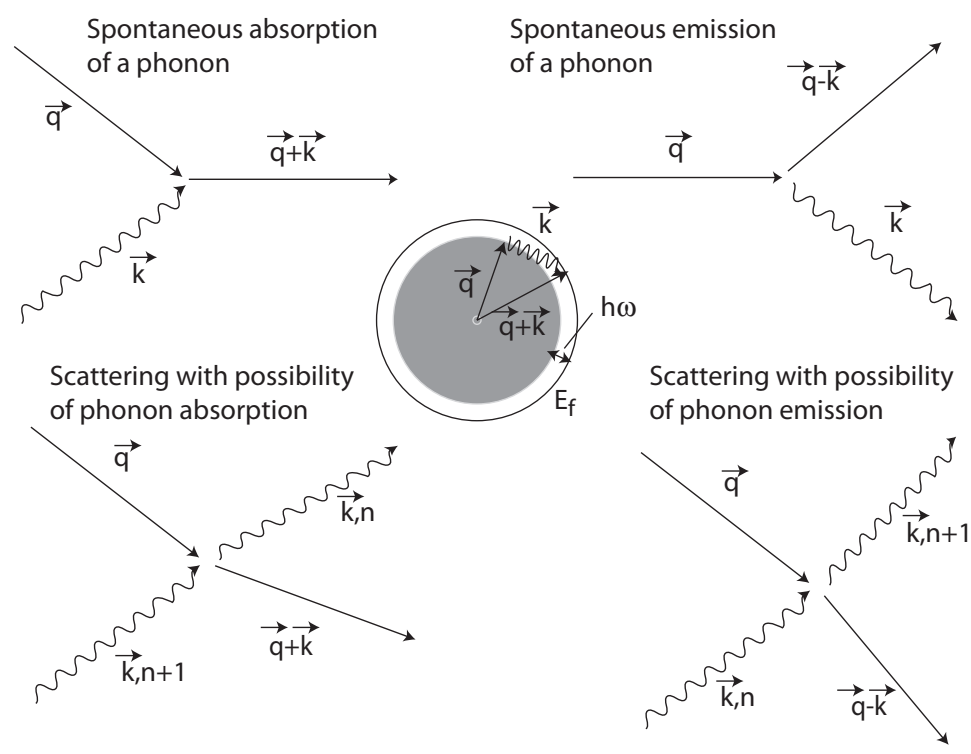

Figure 12. Illustration of electron phonon absorption, emission and scattering processes. The Bloch electrons are represented by straight and the phonons by wavy lines. The number $n$ indicates the occupation level of the phonon state. Crystal momentum has to be conserved for all the processes up to a reciprocal lattice vector. The figure in the middle demonstrates schematically that only electrons in a layer of thickness $\hbar \omega$ around the Fermi surface are concerned by such events due to energy conservation (with $\hbar \omega$ the exchanged phonon energy).

involved. It is equally independent from the harmonic approximation. The other important example is electron-phonon scattering as illustrated in figure 12.

\section{SELECTION RULES}

We would like to finish with a short remark concerning selection rules. Very often in solid state physics we are confronted with problems that have to be treated in perturbation theory. We then have to determine the transition probabilities

$$
<\psi_{\text {final }}\left|H_{1}\right| \psi_{\text {initial }}>
$$

induced by the perturbing Hamiltonian $H_{1}$. The perturbation may be an external field or an internal interaction term that is added to the unperturbed system described by $H_{0}$. The perturbation may either preserve or reduce the symmetry of the system. The wave functions $\mid \psi_{\text {initial }}>$ and $\mid \psi_{\text {final }}>$ are eigenstates of the unperturbed Hamiltonian $H_{0}$. The matrix element $<\psi_{\text {final }}\left|H^{\prime}\right| \psi_{\text {initial }}>$ is by definition a scalar. It thus has to be invariant under all operations of the symmetry group. In other terms, it has to transform according to the fully symmetrical irreducible representation $\Gamma_{1}$. Studying the transformation properties of the matrix element is thus a powerful tool to discriminate between allowed and forbidden transitions.

As we have seen in section 2.1 the eigenstates of $H_{0}$ have to transform according to the irreducible representations of the symmetry group of $H_{0}$. A set of $s^{\alpha}$ functions $\left\{\mid \phi_{j}^{\alpha}>\right\}$ transforms according to the irreducible representation $\Gamma_{\alpha}$ if

$$
T\left(G_{a}\right)\left|\phi_{j}^{\alpha}>=\sum_{l} T_{l, j}^{\alpha}\left(G_{A}\right)\right| \phi_{l}^{\alpha}>\quad \forall G_{a},
$$

with $\left\{T_{l, j}^{\alpha}\left(G_{a}\right)\right\}$ the elements of the matrices of $\Gamma_{\alpha}$. The image of a wave function $\mid \phi_{j}^{\alpha}>$ under the operation $G_{a}$ is thus obtained by multiplying the $j$ th row of the representation matrix $T^{\alpha}\left(G_{A}\right)$ with the 


\section{EPJ Web of Conferences}

vector

$$
\left(\left|\phi_{1}^{\alpha}>, \ldots,\right| \phi_{s^{\alpha}}^{\alpha}>\right)
$$

composed of all the partners in the set. We therefore say that partner $\mid \phi_{j}^{\alpha}>$ transforms according to the $j$ th row of the representation $\Gamma_{\alpha}$.

To characterize the matrix elements we equally have to discuss the symmetry properties of the operator. We have seen in section 2.1 that an operator $S$ transforms under the symmetry elements of the space group according to

$$
S^{\prime}=T\left(G_{a}\right) H T^{-1}\left(G_{a}\right)
$$

The image $S^{\prime}$ of $S$ is in general very different from $S$. If we apply all the symmetry elements of the group to a given operator then we will create a representation of the group. This representation will in general be reducible. In complete analogy with expression (6.2) a set of operators $\left\{S_{i}\left(\Gamma_{\alpha}\right)\right\}$ is said to transform according to an irreducible representation $\Gamma_{\alpha}$ if

$$
S_{i}^{\alpha \prime} \equiv T\left(G_{a}\right) S_{i}^{\alpha} T^{-1}\left(G_{a}\right)=\sum_{k} T_{k, i}^{\alpha}\left(G_{a}\right) S_{k}^{\alpha} \quad \forall G_{a}
$$

The most trivial operator is a constant for which $S^{\prime}=S \quad \forall G_{a}$. It transforms according to the identity representation.

To touch base with the matrix elements we have to investigate the vectors $S \mid \psi>$. Let us label the eigenfunctions of $H_{0}$ according to the irreducible transformations of $H_{0}$

$$
|\psi>=| \phi_{j}^{\alpha}>\text {. }
$$

The subscript $j$ indicates the partner within the irreducible representation $\Gamma_{\alpha}$. We are interested in the symmetry of the image

$$
\left|\psi_{i, j}>=S_{i}^{\alpha}\right| \phi_{j}^{\beta}>
$$

that is produced by an operator of symmetry $\alpha$, when acting on an eigenfunction of symmetry $\beta$. We obtain

$$
\begin{aligned}
T\left(G_{a}\right) \mid \psi_{i, j}> & =T\left(G_{a}\right) S_{i}^{\alpha} \mid \phi_{j}^{\beta}> \\
& =T\left(G_{a}\right) S_{i}^{\alpha} T\left(G_{a}\right)^{-1} T\left(G_{a}\right) \mid \phi_{j}^{\beta}> \\
& =\sum_{k} T_{k, i}^{\alpha}\left(G_{a}\right) S_{k}^{\alpha} T\left(G_{a}\right) \mid \phi_{j}^{\beta}> \\
& =\sum_{k} T_{k, i}^{\alpha}\left(G_{a}\right) S_{k}^{\alpha} \sum_{l} T_{l, j}^{\beta}\left(G_{a}\right) \mid \phi_{l}^{\beta}> \\
& =\sum_{k, l} T_{k, i}^{\alpha}\left(G_{a}\right) T_{l, j}^{\beta}\left(G_{a}\right) S_{k}^{\alpha} \mid \phi_{l}^{\beta}> \\
& =\sum_{k, l} T_{k l, i j}^{\alpha \otimes \beta}\left(G_{a}\right) \mid \psi_{k, l}>,
\end{aligned}
$$

where we have used the identities (6.2) and (6.4) together with the definition (4.26). The set of functions $\left\{\mid \psi_{i, j}>\right\}$ thus transform according to the direct product representations $\Gamma_{\alpha} \otimes \Gamma_{\beta}$.

We now exploit the fact that basis functions transforming according to different irreducible transformations are necessarily orthogonal. Therefore, if $\psi_{\text {final }}$ transforms according to $\Gamma_{\gamma}$ then the matrix element $<\psi_{\text {final }}\left|H_{1}\right| \psi_{\text {initial }}>$ will be zero unless $\Gamma_{\gamma}$ is contained in the decomposition of 


\section{Contribution of Symmetries in Condensed Matter}

$\Gamma_{\alpha} \otimes \Gamma_{\beta}$. This statement can be formulated equivalently as follows: The matrix element

$$
<\phi_{l}^{\gamma}\left|S_{i}^{\alpha}\right| \phi_{j}^{\beta}>
$$

will be zero unless

$$
\Gamma_{\alpha} \otimes \Gamma_{\beta} \otimes \Gamma_{\gamma}
$$

contains the fully symmetrical representation $\Gamma_{1}$. Direct products and related symmetry considerations are of utmost importance in physics. They lead to such important concepts as the Clebsch-Gordon coefficients and the related Wigner-Eckart theorem (see [2]). This goes, however, beyond the scope of this overview.

To give a concrete example for selection rules we discuss the electric dipole operator

$$
H_{1}^{\text {infrared }}=-\vec{E} \cdot \vec{u}
$$

that describes the interaction of a molecule with infrared radiation. As we can treat the electromagnetic field $\vec{E}$ classically only the displacement operator $\vec{u}$ acts on the Hilbert space of vibrations and is thus concerned by the symmetry operations. The perturbation Hamiltonian $H_{1}^{\text {infrared }}$ thus transforms like a vector.

We now ask ourselves the question whether infrared radiation can excite the vibrations of the water molecule, which we had treated in section 4.4. We assume that the initial state of the molecule is a vibrational ground state that has the full symmetry. It thus will transform according to the irreducible representation $A_{1}$ of the group $C_{2 v}$. We already have seen in section 4.4 that a vector transforms according to $A_{1}+B_{1}+B_{2}$. The same decomposition thus applies to the displacement operator

$$
\vec{u} \rightarrow A_{1}+B_{1}+B_{2} .
$$

The presence of three different irreducible representations in the decomposition of $\vec{u}$ underlines the fact that the components $(x, y, z)$ transform differently under the symmetry elements of the water molecule. When $\vec{u}$ acts on the ground state $\left|\psi_{\text {initial }}\right\rangle$ then the image

$$
H_{1}^{\text {infrared }} \mid \psi_{\text {initial }}>
$$

of that operation is transforming according to the direct product

$$
\left(A_{1}+B_{1}+B_{2}\right) \otimes A_{1}=A_{1}+B_{1}+B_{2} .
$$

The vibrations of the water molecule transform according to

$$
2 A_{1}+B_{1} \text {. }
$$

They are thus all a priori infrared active. This is immediately plausible from the fact that all three vibrations correspond to dipole oscillations. In the same way we may deduce that the optic modes of the $\mathrm{NaCl}$ crystal (see section 5.5) at the zone center are infrared active.

If the light is polarized then the selection becomes more stringent. If $\vec{E} \| \hat{z}$, i.e. if $\vec{E}$ points along the two-fold symmetry axis of the molecule then the operator $H_{1}^{\text {infrared }}$ will transform like the $z$-component of a vector. The corresponding irreducible representation is $A_{1}$ (see Table 2). We thus can excite either of the two $A_{1}$ vibrations. This is again evident on physical grounds. Both of these vibrations induce oscillating electrical dipoles along the two-fold symmetry axis of the molecule. If $\vec{E} \| \hat{x}$, i.e. if $\vec{E}$ lies in the molecular plane and is perpendicular to the two-fold axis, then it transforms according to $B_{1}$. We thus can only excite the asymmetric vibration of symmetry $B_{1}$ that breaks the two-fold symmetry of the molecule. If the electric field $\vec{E} \| \hat{y}$ is perpendicular to the plane then it transforms like $B_{2}$. There is no vibrational mode of that symmetry in the water molecule. Light with this polarization would thus not induce infrared activity. 
Modes do not generally have to be infrared active. It is a general statement of group theory that in crystals with inversion symmetry modes are either infrared or Raman active but never both. This is e.g. the case for $\alpha$-quartz.

\section{CONCLUSIONS}

We have in this short overview article tried to demonstrate the power of symmetry arguments when it comes to describe electronic states and ionic excitations in solid state physics. The aim was never to be exhaustive but to give a certain idea of the breadth of the subject. The main impact of symmetry is certainly the characterization of electrons in terms of a band structure and the description of lattice excitations in terms of phonon dispersion relations for periodic solid state systems. These questions thus have hopefully been dealt with the necessary rigor. The concepts elaborated in the context of the lattice modes are by nature very similar to those required for the study of symmetry reduction in structural phase transitions. The reader can verify this statement by consulting the relevant chapters in this book. Other aspects of symmetry like the influence of space group operations on the phonon dispersion relations have been touched upon, however, without discussing all the subtleties (multiplier representations, time inversion symmetry, etc.). Important applications of group theory to solid state physics have been completely neglected. The reader will find all the missing information in the cited literature.

\section{References}

[1] M.S. Dresselhaus, G. Dresselhaus, and A. Jorio, Group Theory, Application to the Physics of Condensed Matter, Springer, Berlin (2008).

[2] J.P. Elliot, P.G. Dawber, Symmetry in Physics, Vol 1 and Vol. 2, Oxford University Press, New York (1984).

[3] Götz Eckold, Symmetry Aspects of Excitations, Phonons in the International Tables of Crystallography, Vol. D, chapter 2.1.

[4] A.A. Maradudin, E.W. Montroll, G.H. Weiss, and I.P. Ipatova, Theory of Lattice Dynamics in the Harmonic Approximation, Academic, New York, (1971)

[5] J.L. Warren, Rev. Mod. Phys. 40, 38 (1968).

[6] Ch. Kittel, Introduction to Solid State Physics, 6th Edition, Wiley (1986), and Physique de l'état solide, Dunod (1998).

[7] N.W. Ashcroft and N.D. Mermin, Solid State Physics, Saunders College, Philadelphia (1976), and Physique des Solides, EDP Science (2002).

[8] M.T. Dove, Structure and Dynamics: an atomic view of materials, Oxford master series in condensed matter physics, Oxford University Press, Oxford (2003).

[9] Felix Bloch, Über die Quantenmechanik der Elektronen in Kristallgittern, Z. Physik 52, 555 (1928).

[10] H. Schober and S. Rols, Les excitations dans la matière condensée, vibrations et phonons, EDP (2010).

[11] K. Schmalzl, D. Strauch, and H. Schober, Phys. Rev. B 68, 144301 (2003).

[12] O.V. Kovalev, Irreducible representations of the space groups, Gordon and Breach, New York (1965).

[13] http://www.cryst.ehu.es/ (Bilbao Crystallographic Server, University of the Basque Country, Bilbao, Basque Country, Spain.)

[14] S.C. Miller and W.H. Love, Tables of Irreducible Representations of Space Groups and CoRepresentations of Magnetic Space Groups, Pruett Press, Denver (1967).

[15] T.G. Worlton and J.L. Warren, Computer Physics Communications 3, 88 (1972). 


\section{Contribution of Symmetries in Condensed Matter}

[16] G. Eckold, UNISOFT - A Program Package for Lattice Dynamical Calculations: Users Manual, Report JÜL 2639 (1992), ISSN 0366-0885; http://www.uni-pc.gwdg.de/Eckold/unisoft.html; G. Eckold, M. Stein-Arsic, H.-J. Weber, J. Appl. Crystallogr. 20, 134 (1987).

[17] G. Raunio, L. Almqvist, and R. Stedman, Phys. Rev. 178, 1469 (1969).

[18] D. Strauch and B. Dorner, J. Phys.: Condens. Matter 5, 6149 (1993).

[19] H. Schober, D. Strauch, K. Nützel, B. Dorner, J. Phys.: Condens. Matter 5, 6155 (1993).

[20] J.M. Perez-Mato, M. Aroyo, J. Hlinka, M. Quilichini, and R. Currat, Phys. Rev. Lett. 81, 2462 (1998). 